\title{
Antcin-H Isolated from Antrodia cinnamomea Inhibits Renal Cancer Cell Invasion Partly through Inactivation of FAK-ERK-C/EBP- $\beta /$ c-Fos-MMP-7 Pathways
}

\author{
Kun-Yuan Chiu, ${ }^{1,2,3}$ Tzu-Hsiu Chen, ${ }^{4}$ Chi-Luan Wen, ${ }^{5}$ Jin-Mei Lai, ${ }^{6}$ Chi-Chih Cheng, \\ Hsiang-Chun Liu, ${ }^{7}$ Shih-Lan Hsu, ${ }^{3,7}$ and Yew-Min Tzeng ${ }^{1,8}$ \\ ${ }^{1}$ Institute of Biochemical Sciences and Technology, Chaoyang University of Technology, Taichung, Taiwan \\ ${ }^{2}$ Division of Urology, Department of Surgery, Taichung Veterans General Hospital, Taichung, Taiwan \\ ${ }^{3}$ Department of Applied Chemistry, National Chi Nan University, Puli, Nantou, Taiwan \\ ${ }^{4}$ Department of Health and Nutrition, Chia Nan University of Pharmacy \& Science, Tainan, Taiwan \\ ${ }^{5}$ Taiwan Seed Improvement and Propagation Station, Council of Agriculture, Propagation Technology Section, Taichung, Taiwan \\ ${ }^{6}$ Department of Life Science, Fu-Jen Catholic University, New Taipei City, Taiwan \\ ${ }^{7}$ Department of Medical Research, Taichung Veterans General Hospital, Taichung, Taiwan \\ ${ }^{8}$ Department of Life Science, National Taitung University, Taitung, Taiwan
}

Correspondence should be addressed to Shih-Lan Hsu; hsu2326@gmail.com and Yew-Min Tzeng; ymtzeng@nttu.edu.tw

Received 1 June 2017; Revised 29 September 2017; Accepted 9 October 2017; Published 2 November 2017

Academic Editor: Jesus R. R. Amado

Copyright (c) 2017 Kun-Yuan Chiu et al. This is an open access article distributed under the Creative Commons Attribution License, which permits unrestricted use, distribution, and reproduction in any medium, provided the original work is properly cited.

Antcin-H, a natural triterpene, is purified from a famous anticancer medicinal mushroom, Antrodia cinnamomea, in Taiwan. This study showed that antcin-H inhibited the growth of human renal carcinoma 786-0 cells; the $\mathrm{IC}_{50}$ value (for $48 \mathrm{~h}$ ) was $170 \mu \mathrm{M}$. Besides, the migration and invasion of 786-0 cells were suppressed by antcin- $\mathrm{H}$ under noncytotoxic concentrations $(<100 \mu \mathrm{M})$; these events were accompanied by inhibition of FAK and Src kinase activities, decrease of paxillin phosphorylation, impairment of lamellipodium formation, and upregulation of TIMPs and downregulation of MMPs, especially MMP-7 expression. Luciferase reporter assay showed that antcin-H repressed the MMP-7 promoter activity, in parallel to inhibiting c-Fos/AP-1 and C/EBP- $\beta$ transactivation abilities. Moreover, antcin-H suppressed the activity of ERK1/2 and decreased the binding ability of C/EBP- $\beta$ and cFos on the upstream/enhancer region of MMP-7 promoter. Overall, this study demonstrated that the anti-invasive effect of antcin- $\mathrm{H}$ in human renal carcinoma 786-0 cells might be at least in part by abrogating focal adhesion complex and lamellipodium formation through inhibiting the Src/FAK-paxillin signaling pathways and decreasing MMP-7 expression through suppressing the ERK1/2$\mathrm{AP}-1 / \mathrm{c}-\mathrm{Fos}$ and C/EBP- $\beta$ signaling axis. Our findings provide the evidence that antcin- $\mathrm{H}$ may be an active component existing in A. cinnamomea with anticancer effect.

\section{Introduction}

Human renal cell carcinoma (RCC), the second common but most lethal cancer of urologic origins, is relatively rare compared to the other carcinomas, but the incidence of RCC is increasing [1]. Although RCC is curable when it is diagnosed in the very early stage of the disease [2], due to its asymptomatic clinical course, by the time of diagnosis about $25 \%$ of RCC patients present with invasion of the tumor to the surrounding tissues and distant metastasis $[3,4]$. New treatment modalities including immunotherapies with interferon or interleukin-2 and targeting therapies focusing on vascular endothelial growth factor and mTOR pathway have been developed recently for the patients with metastatic diseases $[5,6]$. Nevertheless, relatively higher costs and unpredictable side effects limit the clinical uses of all these potential treatment options. Nowadays, no agent can be clinically used to prevent or treat the metastatic RCC and 
most patients ultimately succumb to metastatic disease $[7,8]$. Therefore, an effective therapeutic strategy is a critical issue in the management of these patients.

Cancer metastasis is complex and complicated process that involves several classes of proteins, including adhesion molecules and extracellular proteases. Classical and important metastatic proteins are matrix metalloproteinases (MMPs); numerous reports show that MMPs are overexpressed in metastatic human tumors [9-12]. MMPs can degrade extracellular matrix components and their overexpression correlates with metastasis and poor prognosis in most tumor types $[13,14]$. Among the MMPs, MMP-7, known as matrilysin, has a broad spectrum of proteolytic activity capable of cleaving various types of extracellular matrix [15]. Strong correlations between MMP-7 overexpression and invasion are observed in cancer cell lines and mouse models across most tumor types $[16,17]$. It has been demonstrated that MMP-7 is preferentially expressed at the invasive front of tumors [11, 18], and its overexpression associates with metastatic disease and unfavorable outcome in RCC $[11,12]$. Overall, these findings make MMP-7 a strong and novel target for pharmacological antimetastasis therapy in RCC.

Fungi provide a huge resource and have been used for an effective way to develop new pharmaceutical products. Several studies have shown the potentials of Antrodia cinnamomea, a well-known medical mushroom in Taiwan, on prevention and treatment of liver diseases, food and drug intoxication, hypertension, and cancers [19]. Several compounds have been isolated and identified from fruiting bodies of A. cinnamomea including benzenoids, steroids, and triterpenoids $[19,20]$. The precise compounds and their mode of actions responsible for the observed biological functions have been studied recently [21-24]. Antcins, steroid-like compounds, exert anti-inflammatory effect and enhance blood circulation [25]. A recent report demonstrates that antcin $\mathrm{K}$ triggers intrinsic apoptotic cell death through the mitochondrial and endoplasmic reticulum stress-induced signaling pathways [26]. However, there is no study exploring the effects of antcin- $\mathrm{H}$, a pure compound isolated from $A$. cinnamomea, on human cancer cells. This study was aimed to examine the anticancer effect and its molecular mechanism of antcin-H in human RCC cells. Our results firstly showed that antcin-H inhibited the Src/FAK/paxillin and Src/FAK/ERKc-Fos-C/EBP- $\beta$ signaling pathways to impair lamellipodium formation and decrease MMP-7 expression, consequently suppressing RCC cell migration and invasion, suggesting that antcin-H might have the potential for treating metastatic RCC.

\section{Materials and Methods}

2.1. Isolation of Antcin-H. Antcin-H used in this study was provided by Professor Yew-Min Tzeng at Natural Products and Bioprocess Laboratory, the Institute of Biochemical Sciences and Technology, Chaoyang University of Technology, Taiwan. In brief, the natural product antcin-H was isolated from the cultivated fruiting bodies of $A$. cinnamomea YMT 1002 (GenBank KJ704843). The powdered fruiting bodies (30 g) were extracted through a serial solvent extraction and silica gel column chromatography operations; $65 \mathrm{mg}$ yellow needle antcin-H was obtained with yield of $0.21 \%(\mathrm{~W} / \mathrm{W})$. All the $1 \mathrm{H}$ and 13C NMR spectral data derived from antcin$\mathrm{H}$ were in complete accord with the assigned structure. The isolation method of the natural product antcin- $\mathrm{H}$ was reported by Tzeng's laboratory previously [27]. The purity (>95\%) of antcin-H was confirmed by HPLC analysis [28].

2.2. Cell Culture and Viability Assay. Renal carcinoma 7860 cell line was cultured in Roswell Park Memorial Institute 1640 medium (RPMI 1640) supplemented with 10\% fetal bovine serum, $2 \mathrm{mM}$ L-glutamine, $1 \mathrm{mM}$ sodium pyruvate, $1 \%$ nonessential amino acid, and $1 \mathrm{mM}$ HEPES. Cells were incubated in $95 \%$ air, $5 \% \mathrm{CO}_{2}$ humidified atmosphere at $37^{\circ} \mathrm{C}$. Cell viability was performed with trypan blue exclusion method. Briefly, 786- 0 cells plated in 12 -well plate and treated with various dosages of antcin- $\mathrm{H}(0,20,50,100,200$, and $300 \mu \mathrm{M}$ ) for indicated times. The viable cells were determined by trypan blue reagent.

2.3. Wound-Healing Assay in Live Cells. The in vitro woundhealing assay was performed by using IBIDI culture-insert (IBIDI, Martinsried, Germany) to create a defined cell gap. Briefly, without or with antcin-H treatment, 786-0 cells were trypsinized and seeded in the wells of the cultureinsert containing 10\% serum medium and grown until $95 \%$ confluence. For the cell culture, a linear wound was then created and treated without or with antcin- $\mathrm{H}$ in a low serum condition; to obtain migrating live cell imaging in wounded region, the Olympus microscope was used with a $40 \mathrm{x}$ objective. The image of cell migration into the wound front was microphotographed every $10 \mathrm{~min}$ for up to $24 \mathrm{~h}$.

2.4. Migration and Invasion Assay. With or without antcin$\mathrm{H}$ treatment, 786-0 cells were trypsinized and loaded in the upper chamber of the Transwell apparatus (pore size: $8 \mu \mathrm{m}$; Millipore, Billerica, MA, USA). After treatment, the upper chamber cells were scraped off, the filters were then washed and fixed and stained with Giemsa solution, and then the migrated cells were counted. For invasion assay, cells were loaded onto a matrigel (BD Biosciences, San Jose, CA, USA) precoated Transwell at a density of $2 \times 10^{4}$ cells with serumfree medium, whereas RPMI containing $10 \%$ FBS was added to lower chamber as a chemoattractant. After incubation, the cells that invaded across the matrigel to the lower surface of membrane were fixed in methanol and stained with Giemsa solution, and then the invaded cells were measured.

2.5. Western Blot Analysis. Cells were harvested by scrapping with iced cold PBS and lysed directly in RIPA buffer containing $50 \mathrm{mM}$ Tris- $\mathrm{HCl}(\mathrm{pH} 7.4), 150 \mathrm{mM} \mathrm{NaCl}, 1 \%$ Triton X-100, 0.25\% sodium deoxycholate, $5 \mathrm{mM}$ EDTA ( $\mathrm{pH}$ 8.0 ), and $1 \mathrm{mM}$ EGTA and supplemented with protease and phosphatase inhibitors at $4^{\circ} \mathrm{C}$ for $20 \mathrm{~min}$ and then centrifuged with $12000 \mathrm{rpm}$ at $4^{\circ} \mathrm{C}$ for $30 \mathrm{~min}$ and total protein content was determined by Bradford assay. Equal amounts of total proteins were separated by SDS-polyacrylamide gel electrophoresis. Immunoblotting was performed using primary antibodies against Src, phosphorylated-Src (p-Src), paxillin, 
and phosphorylated-paxillin (p-PXN) (Cell Signaling Technology, Beverly, MA) and ERK1/2, phosphorylated-ERK1/2 (p-ERK1/2), c-Fos, C/EBP- $\beta$, phosphorylated-C/EBP- $\beta$ (pC/EBP- $\beta$ ), FAK, and phosphorylated-FAK (p-FAK) (Santa Cruz, CA, USA). The image was investigated using an ECLPlus detection kit (PerkinElmer Life Sciences, Inc., Boston, MA, USA).

2.6. Immunofluorescence Assay. Cells were cultured on $15 \mathrm{~mm}$ microscope cover glasses in 12-well plate. After antcin- $\mathrm{H}$ was exposed for indicated time, the cells were fixed with $4 \%$ paraformaldehyde and permeabilized by $0.1 \%$ Triton X-100 at room temperature. Samples were blocked with $1 \%$ BSA for $30 \mathrm{~min}$ and incubated with primary antibodies against phosphorylated-FAK and phosphorylatedpaxillin overnight. After being washed with PBS, the cells were incubated with Alexa-Fluor 488-conjugated secondary antibody (Invitrogen, Carlsbad, CA, USA), and then the actin image was investigated using Phalloidin-iFluor 647 Conjugate (AAT Bioquest ${ }^{\circledR}$, Inc., CA) and, finally, incubated with DAPI (Molecular Probes Inc., Eugene, Oregon, USA) for nucleus detection. The cells were then examined using a laser confocal microscope (FV1000, Olympus).

2.7. Quantitative-PCR and RT-PCR. 786-0 cells were exposed to antcin-H or control solvent for indicated time and total RNA was extracted by using TRIzol ${ }^{\circledR}$ isolated kit (Invitrogen, Carlsbad, CA, USA) following the manufacturer's instruction. One $\mu \mathrm{g}$ of total RNA was reverse-transcribed using the First-Strand cDNA Synthesis kit (Thermo Fisher Scientific Inc.). The cDNA products of RT-PCR were then operated with ABI PRISM 7900 Sequence Detector System to determine the mRNA levels according to the manufacturer's instructions. The quantitative RT-PCR reaction mixture contains cDNA, primers, and SYBR Green PCR master mix (Applied Biosystems, Life Technologies). $\beta$-Actin was used as an internal loading control. The used primer sequences were listed as follows: MMP-2: forward $5^{\prime}$-CTTCCAAGTCTGGAGCGATGT-3', reverse 5'-TACCGTCAAAGGGGTATCCAT-3'; MMP-3: forward $5^{\prime}$-GAGGCATCCACACCCTAGGTT- ${ }^{\prime}$, reverse $5^{\prime}$-TAGCTACGTCGGTAAAGACTA3'; MMP-7: forward 5'-GGAGGAGATGCTCACTTCGAT3', reverse 5'-AGGAATGTCCCATACCCAAAGA; MMP13: forward $5^{\prime}$-AAGGAGCATGGCGACTTCT-3', reverse $5^{\prime}$-TGGTTCAGGAAAAGC; tissue inhibitors of metalloproteinases 3 (TIMP-3): forward $5^{\prime}$-CAGGACGCCTTCTGCAA- $3^{\prime}$, reverse $5^{\prime}$-CCCCTCCTTTACCAGCTTCTTC$3^{\prime}$; TIMP-4: forward $5^{\prime}$-CACCCTCAGCAGCACATCTG-3', reverse $5^{\prime}$-GGCCGGAACTACCTTCTCACT-3'.

2.8. Chromatin Immunoprecipitation (ChIP) Assay. ChIP assay was conducted as described previously [29]. Briefly, after treatment, cells were collected and fixed with $1 \%$ formaldehyde at $37^{\circ} \mathrm{C}$ for $10 \mathrm{~min}$ then treated with glycine to quench the cross-links. Cells were harvested by ice PBS containing proteinase inhibitor and lysed with SDS lysis buffer and then sonicated with the following condition: $20 \%$ amplitude for 10 seconds and rest for 10 seconds, repeated 5 times. Lysates were preincubated with the salmon sperm
DNA-protein A agarose (Millipore, Billerica, MA, USA) and subjected to immunoprecipitation overnight at $4^{\circ} \mathrm{C}$ with normal IgG or antibody against C/EBP- $\beta$ (Cell Signaling Technology, Beverly, MA) or c-Fos (Santa Cruz, CA, USA). Precipitates were washed and eluted. The chromatin extracted and protein-DNA cross-links reversed by $\mathrm{NaCl}$. Then DNA was purified by DNA clean-up purification kit (Promega, Madison, WI, USA), and the relative amount of DNA sequence from the MMP-7 promoter region was estimated by PCR analysis. The used primer sequences were listed as follows: for $-1.4 \mathrm{~kb}$ length, $5^{\prime}$-TGAGCTACAGTGGGAACAGG- $3^{\prime}$ and $5^{\prime}$-TCATCGAAGTGAGCATCTCC- $3^{\prime}$; for -876 to -1201 bp region, sense: $5^{\prime}$-CTCCAGCATATTTGGAGTGTTTCC-3' and antisense: $5^{\prime}$-CTTCCAATCACTCTGACTCTGGC-3'; for -263 to $-529 \mathrm{bp}$ region, sense: $5^{\prime}$-CATCTTTCCCCTGTATGGAGAAC- $3^{\prime}$ and antisense: $5^{\prime}$-GACTGCTCTCATAGGTATCATTCAGG-3'; for -138 to $-288 \mathrm{bp}$ region, sense: $5^{\prime}$-CCTGAATGATACCTATGAGAGCAG- $3^{\prime}$ and antisense: $5^{\prime}$-CGAGGAAGTATTACATCGTTATTGG-3'; for +2 to -229 bp region, sense: $5^{\prime}$ GGAGTCAATTTATGCAGCAGACAG- $3^{\prime}$ and antisense: 5'-GGTGTTTTCTGCTAGTGACTGCAG-3'.

2.9. Luciferase Reporter Assay. To generate luciferase reporter, the sequence of MMP-7 containing MluI and BglII restriction site was cloned into the upstream of firefly luciferase gene in PGL3 vector. The primers were +15 to -1000 bp: 5' -ACGCGTTCATTTTTGGTAAGAATGGTCATTGG-3' (forward) and $5^{\prime}$-AGATCTTATGGTTGATTTGGTGTTTTCTGCTAG-3' (reverse). After cloning, the vectors were sequenced to confirm the orientation and integrity of the inserts of each construct. For transfection, cells were cotransfected with the vector DNA and the pRL-CMV internal control which contained the Renilla luciferase gene by Lipofectamine 2000 (Invitrogen, Waltham, MA, USA) and then added with or without antrocin into each well. Cells were collected and analyzed for luciferase activity with the DualLuciferase Reporter Assay System kit (Promega, Madison, WI, USA).

2.10. Statistical Analysis. The results were confirmed by carrying out at least three independent experiments with similar pattern. Values were expressed as means \pm SD from three separate experiments $(n=9)$. Statistical comparisons were done using one-way analysis of variance (ANOVA) with Student's $t$-test, the statistical significance was set at ${ }^{*} p<$ $0.05,{ }^{* *} p<0.01$, and ${ }^{* * *} p<0.001$.

\section{Results}

3.1. Growth Inhibitory Effects of Antcin-H. The chemical structure of antcin-H is shown in Figure 1(a). The effects of antcin-H on renal cancer cell proliferation and human renal carcinoma 786-0 cells were treated with antcin- $\mathrm{H}$. As depicted in Figure 1(b), antcin-H inhibited 786-0 cell growth dose-dependently, and the $\mathrm{IC}_{50}$ value was $170 \mu \mathrm{M}$ after $48 \mathrm{~h}$ exposure. Incubation of 786-0 cells with lower concentrations of antcin-H $(<200 \mu \mathrm{M})$ caused a growth inhibitory effect but did not display any signs of cytotoxicity by morphological 
<smiles>C=C(CC[C@H](C)[C@@]1(C)CC[C@H]2C3=C(C(=O)[C@H](O)[C@@]21C)[C@@]1(C)C(C)C[C@@H](O)[C@H](C)C1CC3=O)C(C)C(=O)O</smiles>

(a)

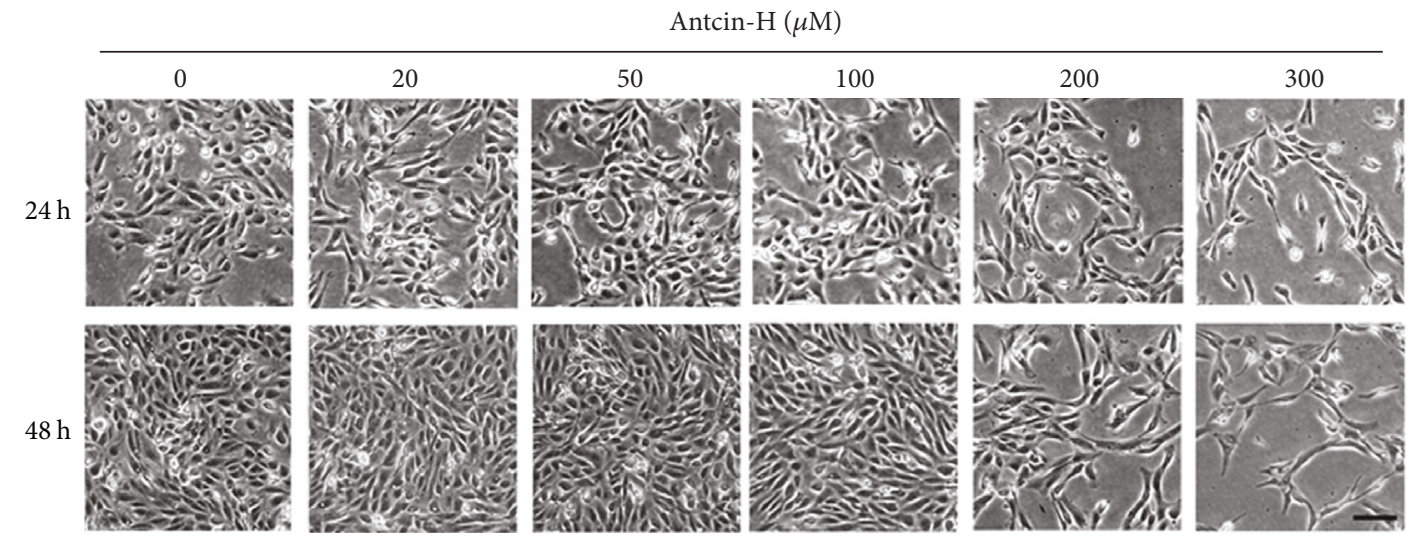

$24 \mathrm{~h}$

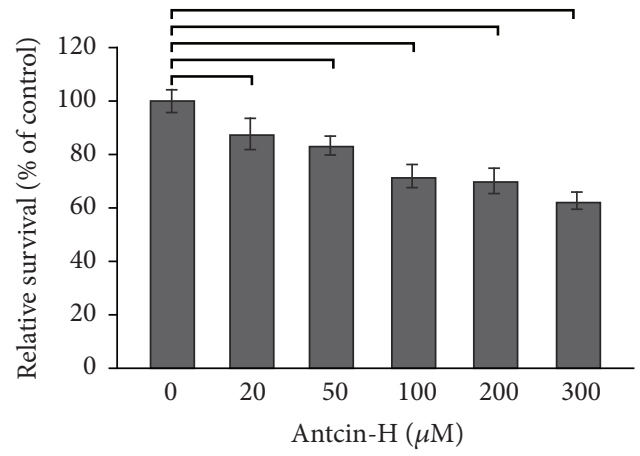

$48 \mathrm{~h}$

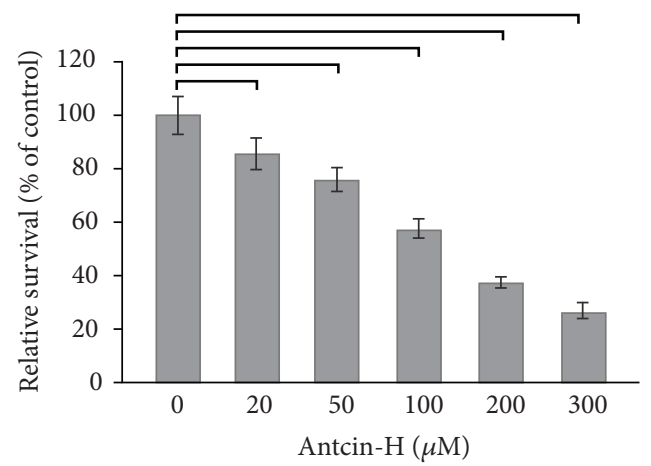

(b)

FIGURE 1: Growth inhibitory effect of antcin-H. (a) The chemical structure of antcin-H. (b) Human RCC 786-0 cells were treated with various concentrations $(0,20,50,100,200$, and $300 \mu \mathrm{M})$ of antcin-H for 24 and $48 \mathrm{~h}$. After incubation, cell morphology was investigated using phasecontrast microscope (upper panel). Scale bar, $100 \mu \mathrm{m}$. The cell viability was determined by trypan blue dye exclusion method (lower panel).

investigation. However, administration of $300 \mu \mathrm{M}$ antcin- $\mathrm{H}$ resulted in rounding and detaching due to cytotoxicity.

\subsection{Inhibition of Migration and Regulation of Migration-} Related Molecules by Antcin- $H$. Metastasis has been considered as a poor prognostic factor in RCC [30]; therefore, developing safe and effective therapeutic agents for the treatment of metastatic RCC is urgently required. To examine the effect of antcin-H on cell migration, Transwell migration assay was carried out. As shown in Figure 2(a), treatment of 786-0 cells with noncytotoxic concentrations of antcin- $\mathrm{H}$ for $24 \mathrm{~h}$ retarded cell migration in a concentration-dependent manner; the number of migrated cells was markedly reduced upon antcin-H treatment.
Previous study demonstrates that FAK/paxillin pathway plays a key role in formation of focal adhesion contact and concomitant cell migration and invasion [31]. Besides, epithelial mesenchymal transition (EMT) is an essential process for cancer cell to acquire migration and invasion ability [32]. Loss of E-cadherin and increase of vimentin are surrogate markers for cell migration and invasion in EMT process. To explore the molecular mechanisms involved in the inhibitory effect of antcin-H on cell migration, the expressed levels of FAK, paxillin, E-cadherin, and vimentin were examined by Western blot analysis. As depicted in Figure 2(b), antcin$\mathrm{H}$ induced a dose-dependent decrease in phosphorylated FAK $^{\text {Tyr925 }}$ and paxillin ${ }^{\text {Tyr118 }}$ levels, with a significant change observed at 50 and $100 \mu \mathrm{M}$, which is consistent with doses 


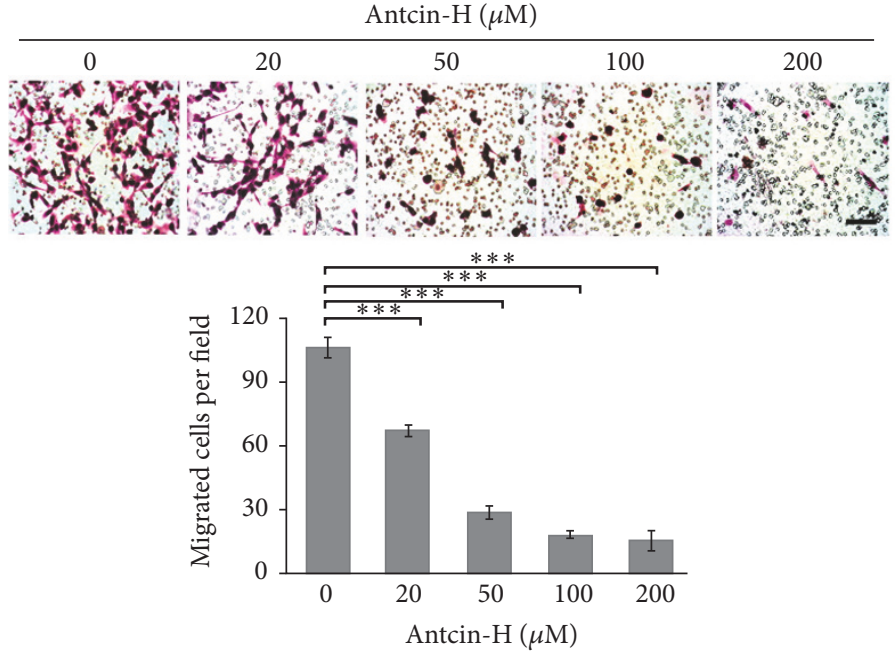

(a)
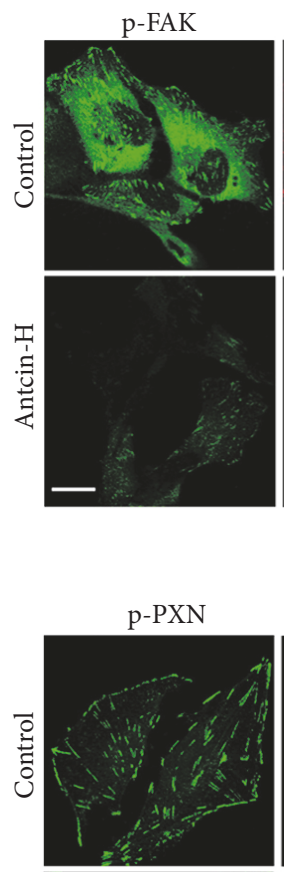
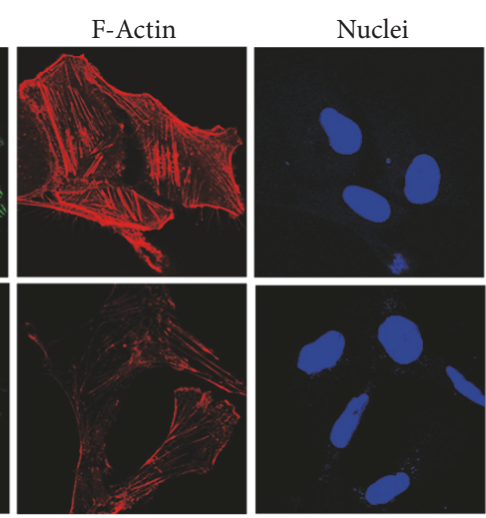

(c)
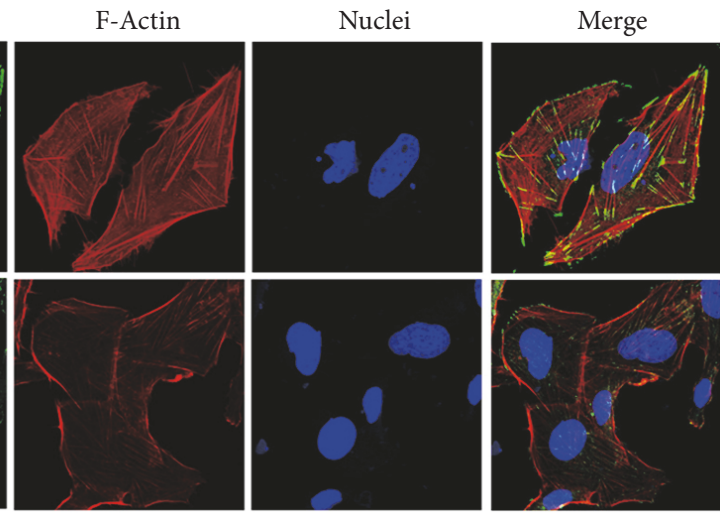

(d)



(b)
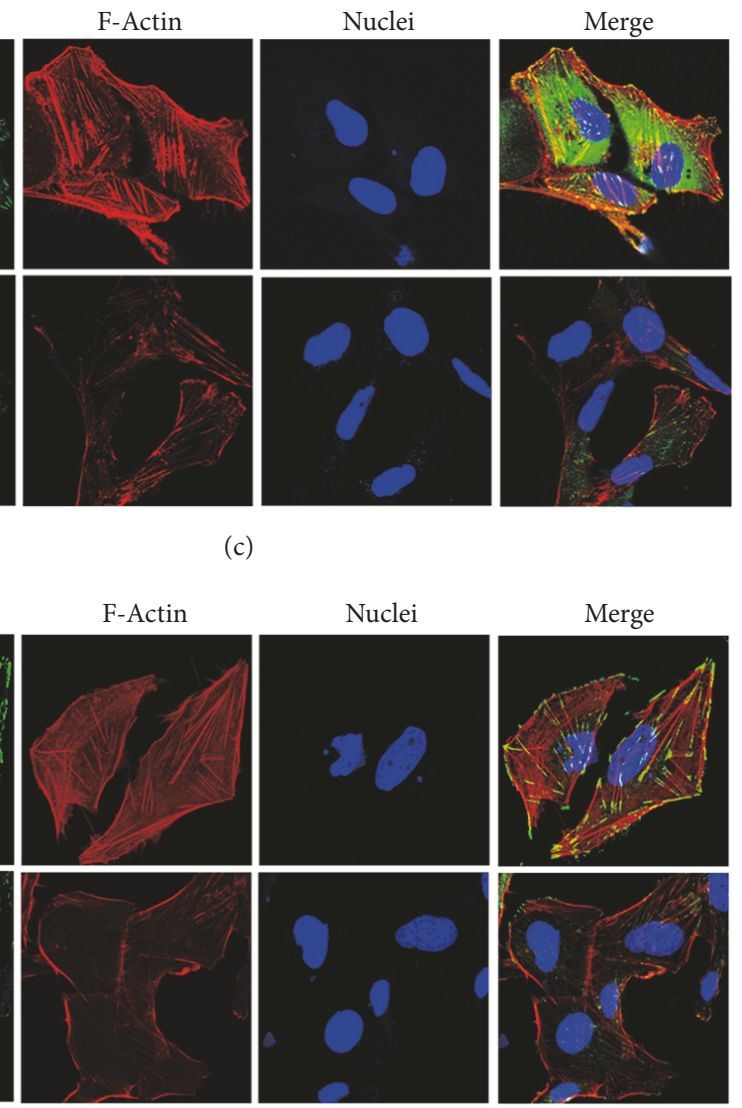

FIGURE 2: Inhibition of cell migration and modulation of migration-related proteins by antcin- $\mathrm{H}$ in vitro. (a) 786-0 cells were treated without or with $20,50,100$, and $200 \mu \mathrm{M}$ antcin-H for $24 \mathrm{~h}$, and then cells were seeded in the upper part of Transwell. After $16 \mathrm{~h}$, cells on the bottom side of the filter were microphotographed and counted. Data were represented as the mean \pm SD of three independent experiments. Statistically significant, ${ }^{* * *} p<0.001$. Scale bar, $100 \mu \mathrm{m}$. (b) Regulation of FAK, paxillin, E-cadherin, and vimentin by antcin-H. 786-0 cells were treated without or with 20,50 , and $100 \mu \mathrm{M}$ antcin-H for $24 \mathrm{~h}$, and then protein lysates were isolated. The levels of phosphorylated-FAK, phosphorylated-paxillin, E-cadherin, and vimentin were examined by Western blot analysis. $\beta$-Actin was used as an internal loading control. Confocal imaging of (c) phosphorylated-FAK and (d) phosphorylated-paxillin. 786-0 cells were treated without or with $100 \mu \mathrm{M}$ antcin- $\mathrm{H}$ for $24 \mathrm{~h}$. The cellular distribution of phosphorylated-FAK and phosphorylated-paxillin was examined by immunofluorescence staining using phosphorylated-FAK and phosphorylated-paxillin specific antibodies. The immunoreactive images were investigated by confocal microscope. Scale bar, $20 \mu \mathrm{m}$. 
that are antimigration (Figure 2(a)). Moreover, the expressed level of vimentin was significantly reduced by antcin- $\mathrm{H}$. However, the levels of total FAK and paxillin were not affected by antcin-H. Moreover, the expression of E-cadherin could not be detected in both control and antcin-H-treated 786-0 cells (Figure 2(b)). These results reveal that FAK, paxillin, and vimentin may be targeting molecules involved in antcin- $\mathrm{H}$ mediated inhibition of 786-0 cell migration.

To further examine the cellular distribution of phosphorylated-FAK and phosphorylated-paxillin, 7860 cells were treated with $100 \mu \mathrm{M}$ antcin-H and subjected to immunostaining analysis with anti-phosphor-FAK and anti-phosphor-paxillin antibody and then counterstained with phalloidin-rhodamine and DAPI for actin and nucleus staining, respectively. Confocal imaging revealed that the majority of control untreated cells showed small dot-like structures of phosphorylated-FAK (Figure 2(c)) and phosphorylated-paxillin (Figure $2(\mathrm{~d})$ ) extending into a polarized, actin-containing lamellipodia. In contrast, antcin-H treatment decreased the phosphorylated-FAK and phosphorylated-paxillin levels at the leading edge and reduced lamellipodia formation in 786-0 cells (Figures 2(c) and $2(\mathrm{~d}))$.

Next, the antimigratory activity of antcin-H was evaluated by real-time, live cell imaging of wound-healing assay. 786-0 cells were incubated with $100 \mu \mathrm{M}$ antcin-H for indicated time periods. In representative time-lapse images, antcin-H treatment significantly retarded cell migratory activity (Figure 3(a)). Because both FAK and paxillin are the important regulators of lamellipodial dynamics in motile cells [33], the formation of lamellipodium at the wound margin was investigated by immunostain with antiphosphorylated-FAK and phosphorylated-paxillin antibody. Small dot-like structures, regarded as FAK (Figure 3(b)) and paxillin (Figure 3(c)) localized at the front edge, which associated with lamellipodium containing bundles of F-actin were detected in untreated 786- 0 cells. Conversely, only a few small dot-like FAK and paxillin immunoreactivity located at front edge of wound margin, and a clear lamellipodia could not be observed in antcin-H-treated 786-0 cells. Since lamellipodia are shown to increase in highly motile cells, these results indicate that antcin- $\mathrm{H}$ exposure may cause a decrease of cell migratory capability in 786-0 cells.

\subsection{Antcin-H Suppresses Cell Invasion and Modulates MMPs} and TIMPs Expression. To examine whether antcin-H could suppress cell invasion in RCC 786-0 cells, Matrigel-coated Transwell invasion assay was conducted, and invaded cells were photographed using a microscope with a $20 \mathrm{x}$ objective lens. Representative images of invaded cells were shown in Figure 4(a), antcin-H effectively impaired the invasive capability of 786-0 cells, and the number of invaded cells was significantly fewer than those in untreated control. At $100 \mu \mathrm{M}$ antcin-H treatment revealed an approximately $80 \%$ decreased invasive ability in comparison with untreated control. Because the expression of MMP and TIMP family members are critical for cancer invasion, to characterize the regulation of MMPs and TIMPs genes by antcin-H, real-time PCR analysis was carried out. Results indicated that MMP-2,
$M M P-3, M M P-7$, and $M M P-13$ genes were downregulated, whereas TIMP-3 and TIMP-4 were upregulated after exposure to antcin-H (Figure 4(b)). However, $M M P-1, M M P-8$, MMP-9, MMP-10, MMP-11, TIMP-1, and TIMP-2 genes were not altered by antcin-H (data not shown).

3.4. Antcin-H Inhibits the ERK Signaling Pathway. To further characterize the possible mechanism that underlies the inhibitory effect of antcin- $\mathrm{H}$ on $786-0$ cells, the activation of several kinases involving FAK pathway and contributing to cell migration and invasion, including Src, FAK, and ERK1/2, was examined by Western blot analysis. As shown in Figure 5(a), the phosphorylated forms of major FAK signaling pathways, including FAK, Src, and ERK1/2, were drastically decreased after exposure to $100 \mu \mathrm{M}$ antcin-H for $4 \mathrm{~h}$ and continuously suppressed at $24 \mathrm{~h}$. Consistently, the major ERK downstream transcription factors, such as c-Fos and phosphorylated-C/EBP- $\beta$, were also time- and dosedependently reduced in response to antcin- $\mathrm{H}$ treatment (Figures 5(a) and 5(b)). However, the amount of total FAK, $\mathrm{Src}$, and ERK1/2 did not change when incubated with antcin$\mathrm{H}$.

3.5. Inhibition of $c-F o s$ and C/EBP- $\beta$ DNA Binding Activity and Transactivation Ability Contributes to Antcin-H-Mediated MMP-7 Downregulation. Growing evidence indicates that MMP-7 is overexpressed in RCC [11] and was clinically associated with metastasis and poor prognosis in patients with RCC $[11,12]$. The present study showed that the expression of MMP-7 gene was unusually reduced following antcin$\mathrm{H}$ administration (Figure 4(b)); to further confirm that the antcin-H-mediated MMP-7 downregulation is regulated at transcriptional level, the promoter activity of MMP-7 was evaluated by the reporter luciferase assay. As depicted in Figure 6(a), antcin-H suppressed reporter activity in 786-0 cells in a concentration-dependent manner. These data suggest that antcin-H reduced $M M P-7$ gene expression through repression of $M M P-7$ promoter activity.

Notably, there are two c-Fos/AP1 (c-FosRE1, -67 -59 and c-FosRE2, -981 -972) and four C/EBP $\beta$ (C/EBP$\beta R E 1,-55 \sim-50$; C/EBP- $\beta$ RE2, -250 -245; C/EBP- $\beta$ RE3, -457 -451; C/EBP- $\beta \mathrm{RE} 4,-997 \sim-985)$ putative binding sites locate upstream of $M M P-7$ promoter (Figure 6(b)). To determine which binding site associated with antcin-H-mediated inhibition of MMP-7 gene expression, 786-0 cells were incubated without or with $100 \mu \mathrm{M}$ antcin-H for $16 \mathrm{~h}$, and then anti-c-Fos or anti-C/EBP- $\beta$ antibody was used to carry out chromatin immunoprecipitation (ChIP) assay. As shown in Figure 6(c), in untreated 786-0 cells, c-Fos appeared to be binding on two potential c-Fos/AP1 response sites, $\mathrm{c}$ FosRE1 and c-FosRE2, located upstream of $M M P-7$ promoter. The interaction of $\mathrm{c}$-Fos to $\mathrm{c}$-FosRE1 was stronger than that to $\mathrm{c}$-FosRE2. However, antcin-H treatment resulted in a great decrease in $\mathrm{c}$-Fos binding on both sites. Besides, $\mathrm{C} / \mathrm{EBP}-\beta$ appeared to bind only three putative response regions (C/EBP- $\beta \mathrm{RE} 1, \mathrm{C} / \mathrm{EBP}-\beta \mathrm{RE} 2$, and $\mathrm{C} / \mathrm{EBP}-\beta \mathrm{RE} 4)$ in untreated cells, whereas exposure to antcin-H significantly diminished the binding of $\mathrm{C} / \mathrm{EBP} \beta$ on these sites. However, the interaction of $\mathrm{C} / \mathrm{EBP}-\beta$ with $\mathrm{C} / \mathrm{EBP}-\beta \mathrm{RE} 3$ could not 

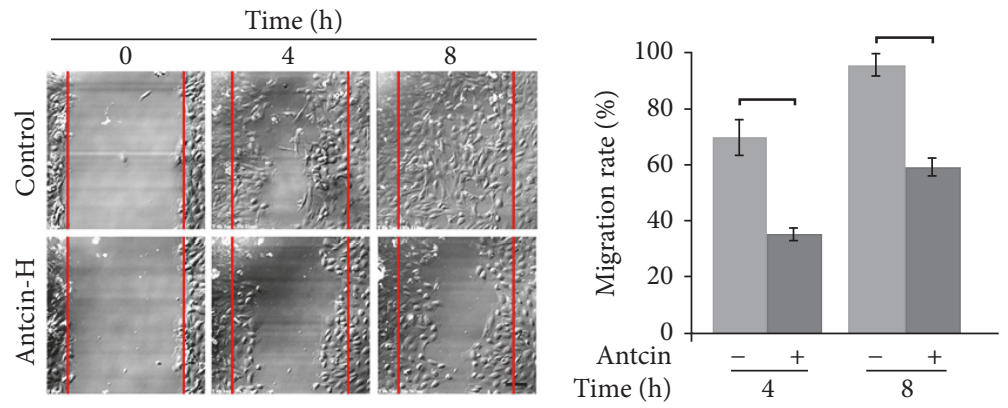

(a)
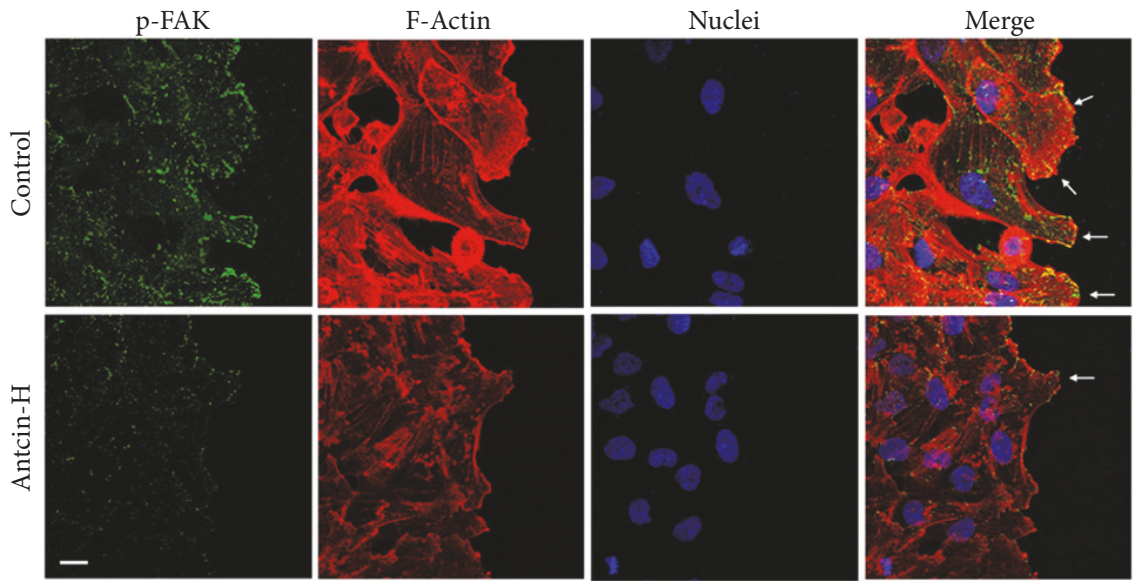

(b)
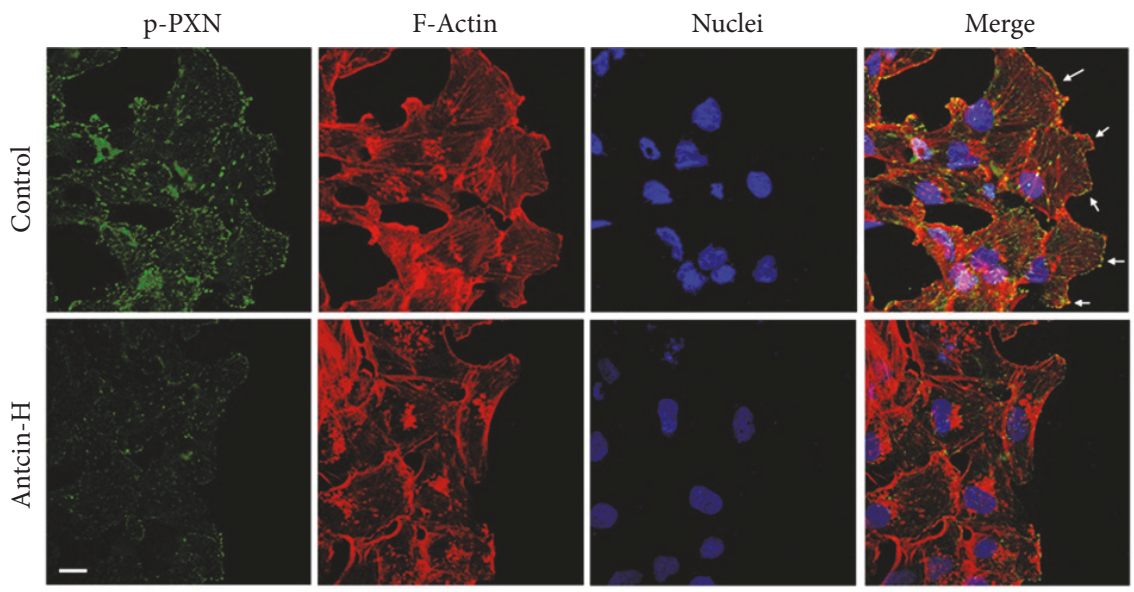

(c)

FIGURE 3: Suppression of wound-healing and disruption of lamellipodium formation by antcin-H. (a) Live cell time-lapse images at woundhealing front. 786-0 cells were cultured to $100 \%$ confluence on glass coverslips. After wound was made, fresh media without or with $100 \mu \mathrm{M}$ antcin- $\mathrm{H}$ were added. Cells were allowed to migrate; the time-lapse of live cell imaging was observed at 4 and $8 \mathrm{~h}$. Scale bar, $20 \mu \mathrm{m}$. (b) Immunostaining with FAK or (c) paxillin antibody. After $8 \mathrm{~h}$ wounding, the migrated cells were fixed, and the immunofluorescence staining was carried out using anti-phosphorylated-FAK and anti-phosphorylated-paxillin antibodies. The immunoreactive image was recorded by confocal microscope. Arrow, formation of lamellipodium. Scale bar, $20 \mu \mathrm{m}$.

be observed in untreated control cells. Moreover, antcin$\mathrm{H}$ dose-dependently blocked c-Fos and C/EBP- $\beta$ binding to their response DNA sequences located at distal and proximal $M M P-7$ promoter region (c-FosRE1, c-FosRE2, C/EBP- $\beta$ RE1, C/EBP- $\beta$ RE2, and C/EBP- $\beta$ RE4), respectively (Figure 6(d)). Additionally, no PCR amplified product was seen in sample which was processed by IgG isotype controlmediated precipitation. These results revealed that antcin- $\mathrm{H}$ could decrease the recruitment of both c-Fos and C/EBP- $\beta$ transcriptional factors into the upstream response elements of $M M P-7$ promoter, ultimately leading to inhibiting the expression of MMP-7 gene in 786-0 RCC cells. 


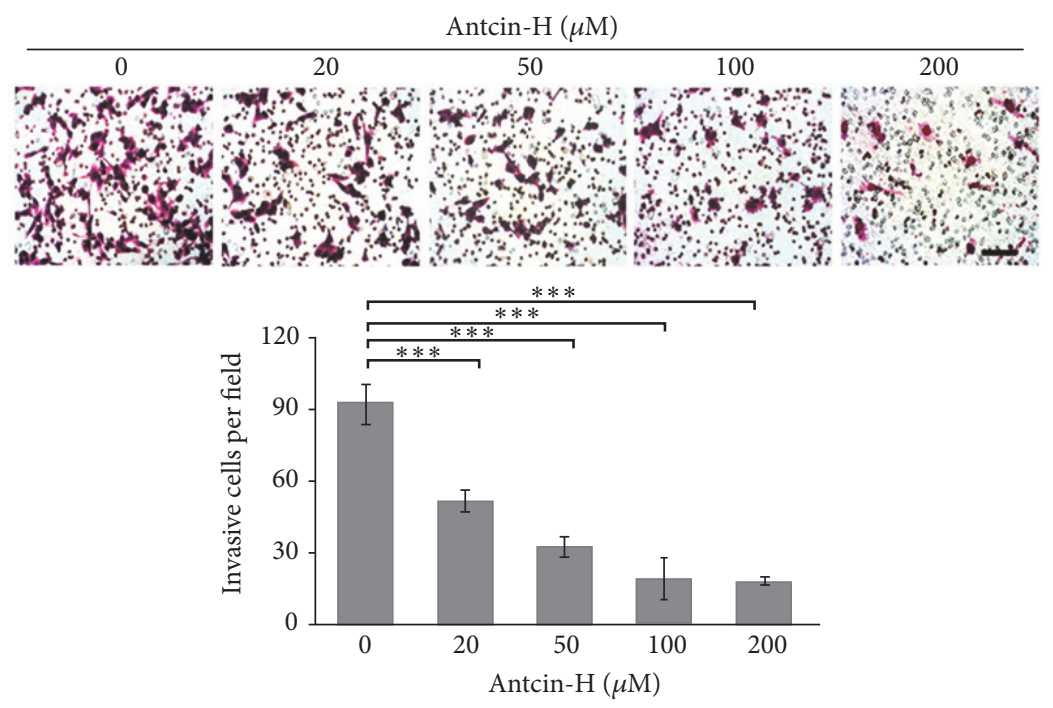

(a)
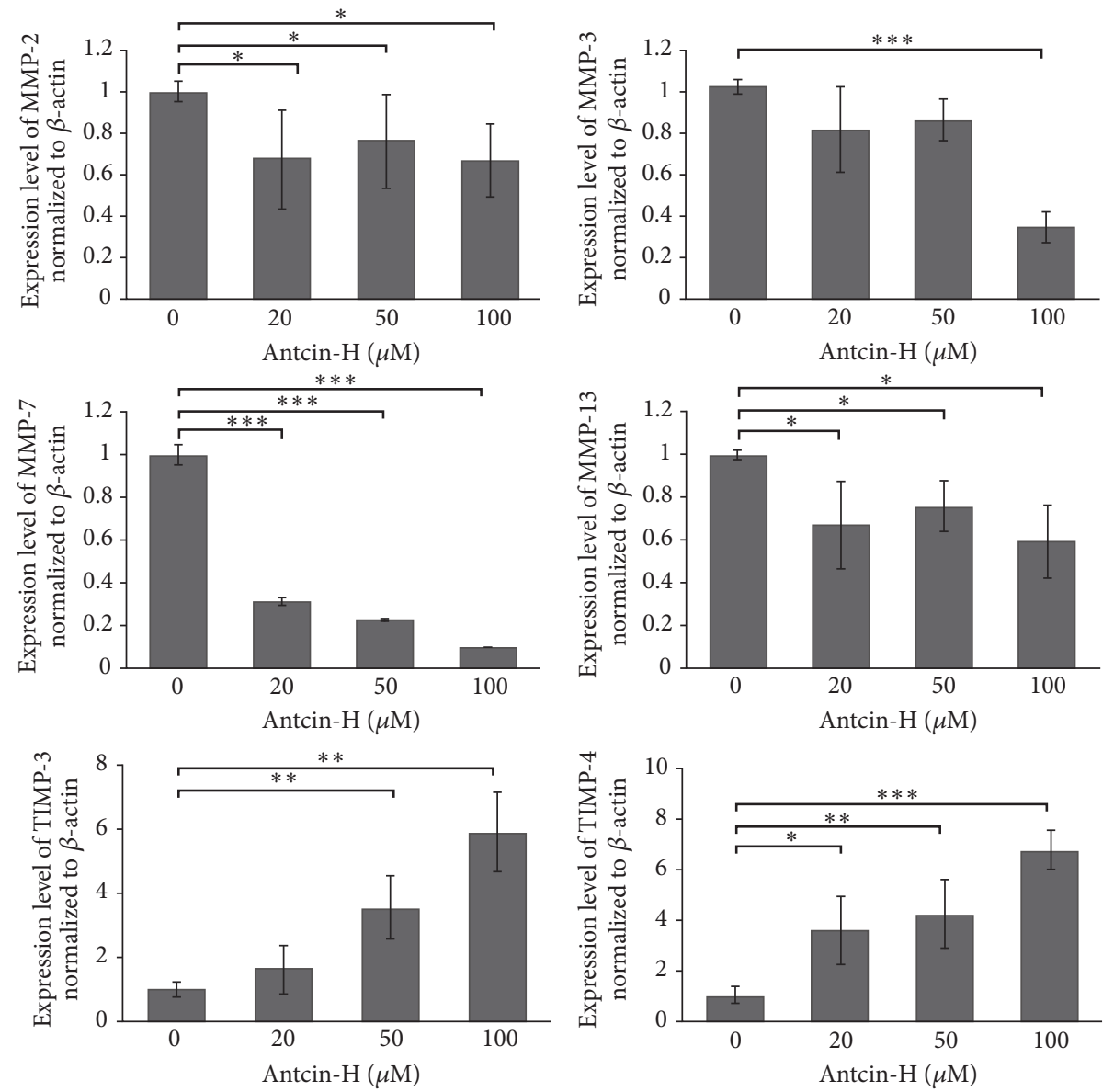

(b)

FiguRE 4: ANTCIN-H prevents invasion and regulates MMPs gene expression. (a) 768-0 cells were pretreated without or with 20, 50, 100, and $200 \mu \mathrm{M}$ antcin- $\mathrm{H}$ for $24 \mathrm{~h}$ and then seeded into Matrigel-coated Transwell apparatus for another $24 \mathrm{~h}$ in the absence or presence of antcin- $\mathrm{H}$. After incubation, the invaded cells were stained with Giemsa solution and counted using a microscope. Scale bar, $100 \mu \mathrm{m}$. The data were represented as the mean \pm SD of nine replicates from three separated experiments. ${ }^{* * *} p<0.001$ versus control. (b) Real-time PCR analysis of MMPs and TIMPs gene expression. Cells were treated without or with 20, 50, and $100 \mu \mathrm{M}$ antcin-H for $24 \mathrm{~h}$. After treatment, the RNA extracted from 786-0 cells was subjected to a real-time PCR. $\beta$-Actin was used as an internal control. Data were represented as the mean \pm $\mathrm{SD}$ of three independent experiments. Statistically significant, ${ }^{*} p<0.05,{ }^{* *} p<0.01$, and ${ }^{* * *} p<0.001$. 


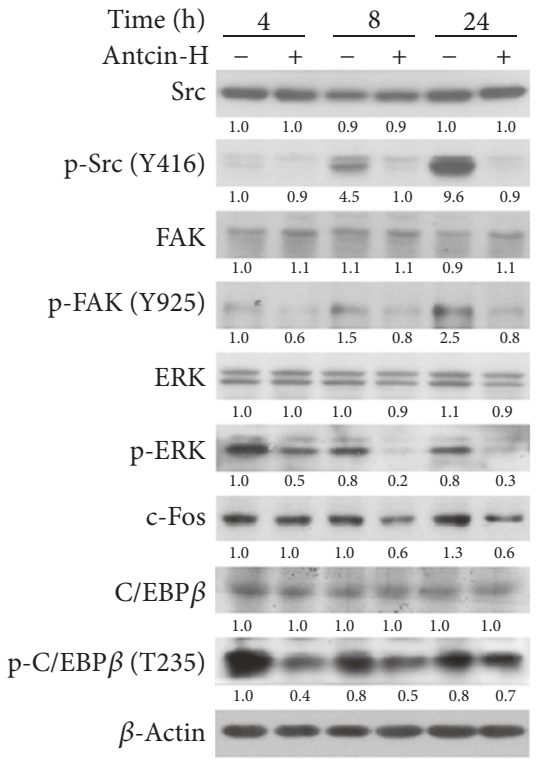

(a)

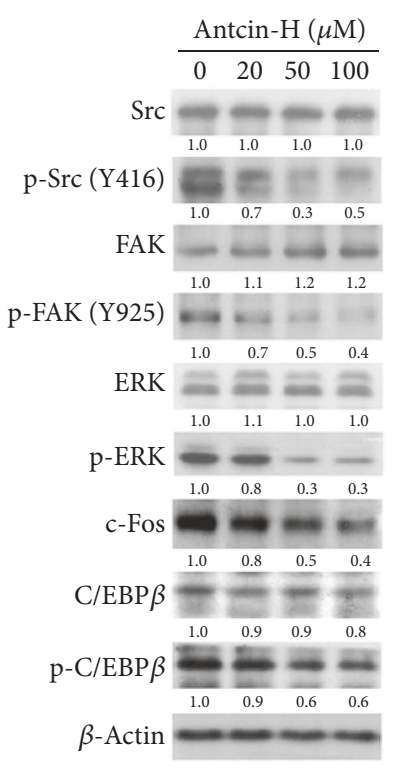

(b)

FIGURE 5: Suppression of Src, FAK, and ERK1/2 signaling pathways by antcin-H. (a) Time course-dependent experiment. 786-0 cells were treated without or with $100 \mu \mathrm{M}$ antcin- $\mathrm{H}$ for 4, 8, and $24 \mathrm{~h}$. (b) Dose-dependent experiment. Cells were treated without or with 20, 50, and $100 \mu \mathrm{M}$ antcin-H for $24 \mathrm{~h}$. After incubation, total protein lysates were isolated; the Western blotting analysis was performed to examine the levels of phosphorylated-Src, phosphorylated-FAK, phosphorylated-ERK1/2, phosphorylated-C/EBP- $\beta$, and c-Fos. $\beta$-Actin was used as an internal loading control.

\section{Discussion}

RCC is an epithelial malignancy of human kidney; surgery is the major strategy for treating patients with RCC. Unfortunately, approximately $30 \%$ of patients with RCC will be diagnosed with metastatic disease. Although target therapy and immunotherapy for treatment of patients with metastatic RCC have shown some positive results, continuous treatments with these drugs are associated with a high incidence of toxic effects and resistance [7], and five-year survival of patients with metastatic RCC is only $10 \%$ [34]. Therefore, developing strategies for therapeutic interventions in metastatic RCC is of utmost importance. Numerous natural substances have been found to inhibit progression and metastasis of various types of cancer cell lines and reveal that they might be useful for the treatment of metastatic cancer [35, 36]. The present study showed for the first time that antcin$\mathrm{H}$, a steroid-like compound isolated from a famous anticancer medicinal mushroom A. cinnamomea, inhibited Src, FAK, and ERK1/2 signaling pathways and thereby decreased phosphorylated-paxillin, phosphorylated-C/EBP- $\beta$, and total c-Fos levels and downregulated vimentin and MMPs expression, finally leading to impaired lamellipodium formation and cellular migration/invasion at nontoxic concentrations in human RCC 786-0 cells.

The FAK-Src complex is a pivotal component of focal adhesion contact, as a critical signaling module controls cell motility and potentiates tumor metastasis [31, 37]. Paxillin localizes at focal adhesion contact and acts as a scaffold molecule providing a platform for FAK and Src, which are involved in cell migration events associated with tumor metastasis [38]. Activated FAK/Src complex phosphorylates cytoskeletal adaptor paxillin which promotes cell migration [39]. FAK-Src expression and function have been associated with the majority of invasive and metastatic human cancers, with poor survival $[40,41]$. Therefore, inhibition of FAK-Src function and expression is considered as a potential strategy for cancer therapy [42]. A synthetic small molecule with anticancer effect inhibits FAK, Src, and paxillin expression and activation, leading to the suppression of cell migration in colon cancer cells [43]. Src inhibitors reduce the migration of several types of human cancer cell through blocking Src, FAK, p130CAS, and paxillin activation [44, 45]. Inhibiting FAK/paxillin signaling by a sialyltransferase inhibitor effectively retards cancer cell migration [46]. In agreement with these earlier reports, this study showed that antcin-H significantly impaired cell migration by suppression of the FAK, Src, and paxillin signaling pathways. Besides, FAK, paxillin, and actin filaments play the important role in lamellipodium formation [33]. Since lamellipodium is a cell protrusion which is critical for directional migration in vary types of cell [47]. Here, we showed that antcin-H-mediated inhibition of FAK-Src-paxillin signaling axis and RCC cell migration was accompanied by decrease of lamellipodium, indicating that disruption of the lamellipodium formation as well as inhibition of migration and wound closure attributed to reducing formation of focal adhesion and actin bundles through suppressing FAK-Src-paxillin signaling pathway in antcin-H-treated cells. 


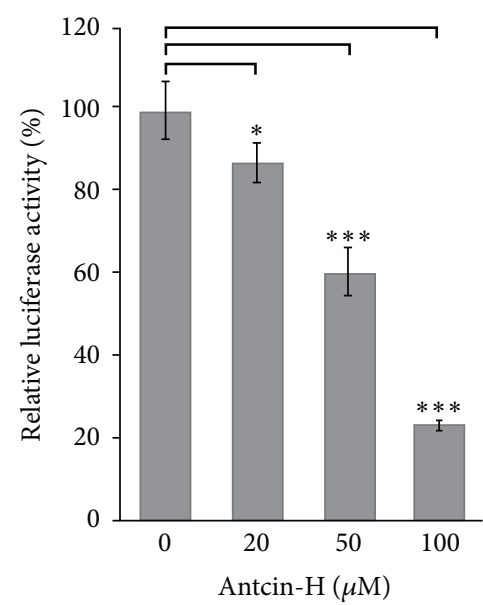

(a)

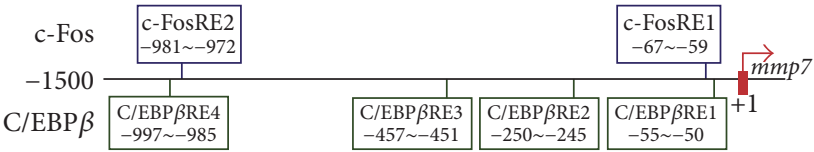

(b)

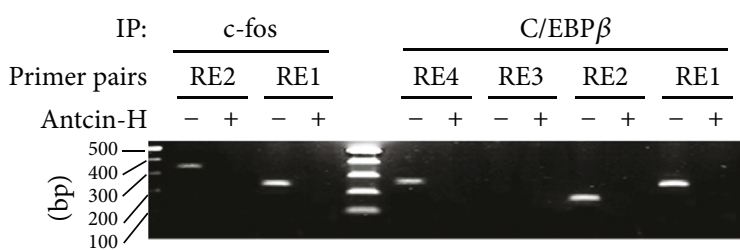

(c)

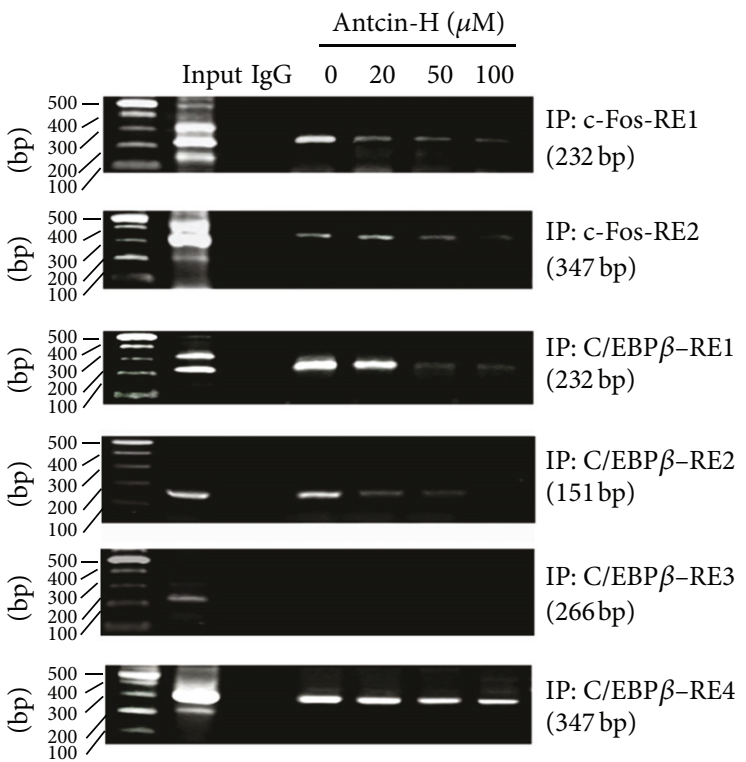

(d)

FIGURE 6: Inhibition of $c$-Fos and C/EBP- $\beta$ activities involved in antcin-H-mediated $M M P-7$ downregulation. (a) Reporter luciferase assay. 786- 0 cells were transiently transfected with reporter vector containing $M M P-7$ promoter $+1 \sim-1500$ region or control vector for $24 \mathrm{~h}$, and then the cells were treated without or with 20,50 , and $100 \mu \mathrm{M}$ antcin- $\mathrm{H}$ for another $24 \mathrm{~h}$. After incubation, the luciferase activity was measured and the relative luciferase activity was presented as means \pm SD. Statistically significant, ${ }^{*} p<0.05,{ }^{* *} p<0.01$, and ${ }^{* * *} p<0.001$. (b) Putative binding sites of c-Fos and C/EBP- $\beta$ located at upstream of MMP-7 promoter. (c) Antcin-H prevents c-Fos and C/EBP- $\beta$ binding to the MMP-7 upstream promoter/response element region. The 786-0 cells were treated without or with $100 \mu \mathrm{M}$ antcin- $\mathrm{H}$ for $24 \mathrm{~h}$, the cells were collected, and then ChIP assay was carried out. (d) Antcin-H dose-dependently inhibits c-Fos and C/EBP- $\beta$ binding to the MMP-7 upstream promoter/response element region. The 786-0 cells were treated without or with 20,50 , and $100 \mu \mathrm{M}$ antcin- $\mathrm{H}$ for $24 \mathrm{~h}$, the cells were collected, and then the activity of c-Fos and C/EBP- $\beta$ binding to each response site located at MMP-7 promoter upstream was determined by ChIP assay.

Growing evidence demonstrates that FAK/ERK-stimulated signaling activates EMT [48]. EMT is an essential step for cancer cell to acquire migration and invasion ability; loss of E-cadherin and increase of vimentin are surrogate markers for cell migration and invasion in EMT process [32]. E-cadherin is a normal epithelial cell adhesion molecule and is considered as a cancer metastasis suppressor. Nevertheless, methylation and loss of heterozygosity of E-cadherin gene are a common event in advanced renal cell carcinoma tissues and cell lines, including 786-0 cell line, which can lead to inactivation of E-cadherin transcription and loss of Ecadherin protein expression [49]. This is consistent with our observation that E-cadherin could not be detected in 7860 cells. On the other hand, evidence supports that vimentin is a crucial cytoskeletal component of motile mesenchymal cells, including epithelium-derived metastatic tumor cells. 
Downregulation of E-cadherin or overexpression of vimentin is strongly associated with metastasis, resulting in poor prognosis [50-52]. In the current study, downregulation of vimentin by antcin- $\mathrm{H}$ suggested that a loss of EMT and inhibition of invasion potential might occur in antcin- $\mathrm{H}-$ treated 786- 0 cells.

Literatures show that the process of tumor growth, invasion, and metastasis is tightly regulated by MMPs in various types of malignant tumors, including RCC [11, 12]. Among MMPs, matrilysin (MMP-7) is mainly expressed in malignant tumor cells and preferentially localized at the invasive front of tumors suggesting that it may facilitate destruction of surrounding extracellular matrix and basement membrane $[11,18,53]$. Previous studies demonstrate that MMP-7 is overexpressed in RCC, and increased MMP-7 expression significantly correlates with the malignant behavior of RCC, including invasion, distant metastasis, poor prognosis, and reduced patient survival $[11,54]$. These findings suggest that MMP-7 might be regarded as important targets for metastatic RCC therapy to prevent tumor progression and improve survival. In the current study, antcin- $\mathrm{H}$ slightly decreased MMP-2, MMP-3, and MMP-13 gene expression while it did not alter the expression of MMP-1, MMP-8, MMP-9, MMP10 , and MMP-11 mRNA levels (data not shown). Intriguingly, the expression of MMP-7 gene was drastically downregulated by antcin- $\mathrm{H}$ in 786-0 cells, revealing that targeted inhibition of MMP-7 gene expression might contribute to impairment of RCC cell invasion upon antcin- $\mathrm{H}$ administration. Previous studies have shown that MMP activities modulate at the levels of transcriptional regulation, enzymatic activation, and TIMPs inhibition [55]. TIMPs are endogenous inhibitors of MMPs, consisting of four members, TIMP-1, TIMP-2, TIMP-3, and TIMP-4, and play crucial roles in several processes, including cell proliferation, invasion and migration, angiogenesis, and apoptosis [56]. Deregulated expression of TIMPs has been implicated in tumor invasion and metastasis [56]. Overexpression of TIMPs has been reported to inhibit invasive and progressive potentials of the tumor cells [57]. Among TIMPs, TIMP-3 is a broad inhibitor against all MMPs [58], while TIMP-4 is MMP2 and MMP9 inhibitor $[56,59]$. Our results indicated that upregulation of TIMP3 and TIMP-4 expression, but not TIMP-1 and TIMP-2, might be another potential mechanism that contributes to inhibiting MMPs function in response to antcin- $\mathrm{H}$ treatment. Besides, TIMP family members have been shown to have proangiogenic effect. Overexpression of TIMP-1 is associated with VEGF expression and promoting neovascularization in breast carcinoma rats [60]. TIMP-2 inhibits angiogenesis by directly binding to $\alpha 3 \beta 1$ integrin $[61,62$ ] or insulin-like growth factor-1 receptor [63]. TIMP-4 is previously reported as a positive regulator of angiogenesis [64, 65]. Unlike TIMP1 and TIMP-4, TIMP-3 inhibits angiogenesis by blocking the binding of VEGF to VEGF receptor-2 [66] and suppressing angiotensin II receptor activity [67]. These results suggest the important functions of TIMPs in cancer metastasis and angiogenesis. However, the roles of TIMP-3 and TIMP-4 induced by antcin-H in RCC progression are still poorly understood. Further in vitro and in vivo studies are needed to examine the effects and molecular mechanisms of antcin- $\mathrm{H}$ on TIMP-3/4 regulation and angiogenesis in metastatic RCC.

Previous reports demonstrate that FAK/ERK signaling is not only critical for cell migration and invasion, but is also involved in the regulation of MMPs activity and gene expression $[68,69]$. ERK and its downstream transcriptional factor AP-1 play an important role in the regulation of MMPs gene expression [70, 71]. AP-1 is a common transcriptional activator composed of the Jun and Fos family members [72]. Besides, ERK can phosphorylate Elk-1 which subsequently upregulates c-Fos gene expression [73]. Literatures have shown that the MMP-7 expression is controlled by modulating the activation of AP-1 transcription factors, c-Fos and c-Jun, through ERK1/2 signaling pathway [17, 74-76]. In this study, we found that two putative binding sites of c-Fos were located at the promoter region of MMP-7 (Figure 6(b)). In agreement with these previous studies, our observations provided evidence that antcin-H-inhibited MMP-7 gene expression might be through suppressing ERK/c-Fos signaling axis in RCC 786-0 cells. In addition to AP-1/c-Fos, our results indicated that antcin-H-mediated MMP-7 gene downregulation was also via C/EBP- $\beta$ transcriptional repression. Previous studies demonstrate that phosphorylatedC/EBP- $\beta$ by ERK can activate its transcriptional activity [77]. Our results demonstrated that antcin-H inhibited MMP-7 expression might be also via prevention of ERK-C/EBP- $\beta$ activation. On the basis of these results, we suggest that the anti-invasive activity of antcin- $\mathrm{H}$ is in part due to the inhibition of MMP-7 expression, which plays a critical role in cancer invasion and metastasis, through the suppression of ERK-mediated AP-1/c-Fos and C/EBP- $\beta$ activities in RCC cells.

In fact, our results demonstrated that, except MMP-7, antcin- $\mathrm{H}$ also inhibited other MMP gene expressions, such as MMP-2, MMP-3, and MMP-13, which play critical roles in cancer cell invasion. Similar to MMP-7, these MMPs were also regulated predominantly at the transcriptional level. On the basis of the composition of cis-regulatory elements in their promoters, MMP-2, MMP-3, and MMP-13, also contain AP- 1 and C/EBP- $\beta$ binding sites proximal and distal to the transcriptional start site, and their expressions have been shown to be regulated at the transcriptional levels via AP1 and C/EBP- $\beta$ [78-82]. It is possible that these MMPs are regulated similarly upon antcin- $\mathrm{H}$ treatment. Besides, on the basis of present results, it is still unclear how TIMP-3 and TIMP-4 expression is upregulated in response to antcin$\mathrm{H}$ treatment. Several transcription factors binding sites, for example, p53, STATs, PPARs, AP-1/c-Fos, C/EBP- $\alpha$, and $\mathrm{C} / \mathrm{EBP}-\beta$, are found in the promoter region of TIMP- 3 and TIMP-4. However, which transcription factor contributes to the upregulation of TIMP-3 and TIMP- 4 by antcin-H needs to be further examined.

\section{Conclusion}

Based on our observation, we propose a potential mechanism in antcin-H-treated RCC cells, which shows that antcin-H suppressed FAK-related signaling pathway ( $\mathrm{Src}$, FAK, paxillin, and ERK1/2), which impaired focal adhesion 


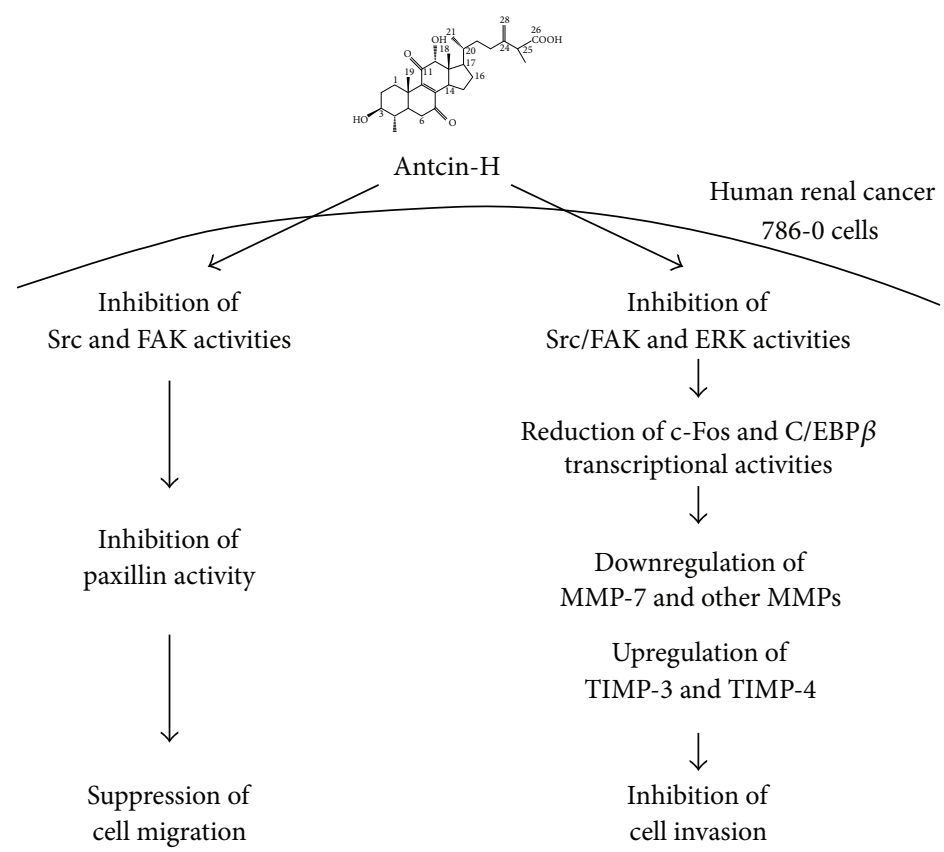

FIGURE 7: Schematic model of the proposed signaling pathways involved in suppressing cell migration and invasion by antcin-H in human RCC 786-0 cells. Antcin-H inhibits Src, FAK, and ERK1/2 phosphorylated activation, in turn decreasing paxillin, c-Fos, and C/EBP$\beta$ activities, reducing the binding of $\mathrm{c}$-Fos and phosphorylated-C/EBP- $\beta$ to AP-1 and C/EBP- $\beta$ response elements, thereby decreasing MMPs gene expression, especially MMP-7. Downregulation of MMP-7 and upregulation of TIMP-3 and TIMP-4 gene expression block the degradation of the extracellular matrix proteins and impair the cell invasion. Besides, reducing paxillin phosphorylation and vimentin expression prevents 786- 0 cell motility.

turnover and lamellipodium formation, inactivated c-Fos and C/EBP- $\beta$, downregulated MMPs (especially MMP-7), and upregulated TIMPs (TIMP-3 and TIMP-4) expression (Figure 7). MMP-7 has been considered as a metastatic marker and survival predictor in RCC patients; inhibition of MMP-7 expression and function in tumor cells could be one of the most powerful strategies in metastatic RCC therapy. Our findings provide new insights into the antimigratory and anti-invasive properties of antcin- $\mathrm{H}$ and implicate that antcin- $\mathrm{H}$ might be a promising phytochemical existing in A. cinnamomea with antimetastatic capability in treating advanced RCC.

\section{Conflicts of Interest}

The authors declare that there are no conflicts of interest regarding the publication of this paper.

\section{Authors' Contributions}

Kun-Yuan Chiu and Tzu-Hsiu Chen have equal contribution.

\section{Acknowledgments}

This study was supported by grants from the National Science Council of Taiwan (MOST 103-2320-B-075A-002-MY3 and NSC 102-2320-B-075A-003) and Taichung Veterans General Hospital (TCVGH-1037305C) for Dr. Shih-Lan Hsu.

\section{References}

[1] Taipei, Department of Health and Welfare, Vital Statistics 2014, http://ghdx.healthdata.org/organizations/ministry-health-andwelfare-taiwan-0.

[2] C. Battagli, R. G. Uzzo, E. Dulaimi et al., "Promoter hypermethylation of tumor suppressor genes in urine from kidney cancer patients," Cancer Research, vol. 63, no. 24, pp. 8695-8699, 2003.

[3] R. C. Flanigan, S. C. Campbell, J. I. Clark, and M. M. Picken, "Metastatic renal cell carcinoma.," Current Treatment Options in Oncology, vol. 4, no. 5, pp. 385-390, 2003.

[4] R. G. Uzzo, E. E. Cherullo, J. Myles, and A. C. Novick, "Renal cell carcinoma invading the urinary collecting system: Implications for staging," The Journal of Urology, vol. 167, no. 6, pp. 23922396, 2002

[5] B. Escudier, T. Eisen, W. M. Stadler et al., "Sorafenib in advanced clear-cell renal-cell carcinoma," The New England Journal of Medicine, vol. 356, no. 2, pp. 125-134, 2007.

[6] B. C. Leibovich, K.-R. Han, M. H. T. Bui et al., "Scoring algorithm to predict survival after nephrectomy and immunotherapy in patients with metastatic renal cell carcinoma: a stratification tool for prospective clinical trials," Cancer, vol. 98, no. 12, pp. 2566-2575, 2003.

[7] M. I. Khan, A. M. Czarnecka, R. Duchnowska, W. Kukwa, and C. Szczylik, "Metastasis-initiating cells in renal cancer," Current Signal Transduction Therapy, vol. 8, no. 3, pp. 240-246, 2014.

[8] C. N. Sternberg, R. E. Hawkins, J. Wagstaff et al., "A randomised, double-blind phase III study of pazopanib in patients with 
advanced and/or metastatic renal cell carcinoma: Final overall survival results and safety update," European Journal of Cancer, vol. 49, no. 6, pp. 1287-1296, 2013.

[9] R. D. Bonfil, S. Chinni, R. Fridman, H.-R. Kim, and M. L. Cher, "Proteases, growth factors, chemokines, and the microenvironment in prostate cancer bone metastasis," Urologic Oncology: Seminars and Original Investigations, vol. 25, no. 5, pp. 407-411, 2007.

[10] E. I. Deryugina and J. P. Quigley, "Matrix metalloproteinases and tumor metastasis," Cancer and Metastasis Reviews, vol. 25, no. 1, pp. 9-34, 2006.

[11] Y. Miyata, T. Iwata, K. Ohba, S. Kanda, M. Nishikido, and H. Kanetake, "Expression of matrix metalloproteinase-7 on cancer cells and tissue endothelial cells in renal cell carcinoma: prognostic implications and clinical significance for invasion and metastasis," Clinical Cancer Research, vol. 12, no. 23, pp. 6998-7003, 2006.

[12] A. Ramankulov, M. Lein, M. Johannsen, M. Schrader, K. Miller, and K. Jung, "Plasma matrix metalloproteinase-7 as a metastatic marker and survival predictor in patients with renal cell carcinoma," Cancer Science, vol. 99, no. 6, pp. 1188-1194, 2008.

[13] A. K. Chaudhary, S. Pandya, K. Ghosh, and A. Nadkarni, "Matrix metalloproteinase and its drug targets therapy in solid and hematological malignancies: an overview," Mutation Research, vol. 753, no. 1, pp. 7-23, 2013.

[14] R. Roy, J. Yang, and M. A. Moses, "Matrix metalloproteinases as novel biomarkers and potential therapeutic targets in human cancer," Journal of Clinical Oncology, vol. 27, no. 31, pp. 52875297, 2009.

[15] U. I. Sires, G. L. Griffin, T. J. Broekelmann et al., "Degradation of entactin by matrix metalloproteinases. susceptibility to matrilysin and identification of cleavage sites," The Journal of Biological Chemistry, vol. 268, no. 3, pp. 2069-2074, 1993.

[16] L. E. Jones, M. J. Humphreys, F. Campbell, J. P. Neoptolemos, and M. T. Boyd, "Comprehensive analysis of matrix metalloproteinase and tissue inhibitor expression in pancreatic cancer: increased expression of matrix metalloproteinase-7 predicts poor survival," Clinical Cancer Research, vol. 10, no. 8, pp. 28322845, 2004.

[17] W. Yue, Q. Sun, R. Landreneau et al., "Fibulin-5 suppresses lung cancer invasion by inhibiting matrix metalloproteinase7 expression," Cancer Research, vol. 69, no. 15, pp. 6339-6346, 2009.

[18] Y. Adachi, H. Yamamoto, F. Itoh, Y. Hinoda, Y. Okada, and K. Imai, "Contribution of matrilysin (MMP-7) to the metastatic pathway of human colorectal cancers," Gut, vol. 45, no. 2, pp. 252-258, 1999.

[19] M. Geethangili and Y.-M. Tzeng, "Review of pharmacological effects of Antrodia camphorata and its bioactive compounds," Evidence-Based Complementary and Alternative Medicine, vol. 2011, Article ID 212641, 17 pages, 2011.

[20] M. Geethangili, Y. K. Rao, and Y. M. Tzeng, "Development and validation of a HPLC-DAD separation method for determination of bioactive antrocin in medicinal mushroom antrodia camphorata," International Journal of Applied Science and Engineering Research, vol. 2, pp. 195-201, 2013.

[21] C.-W. Chang, Y.-S. Chen, C.-C. Chen et al., "Targeting cancer initiating cells by promoting cell differentiation and restoring chemosensitivity via dual inactivation of STAT3 and src activity using an active component of antrodia cinnamomea mycelia," Oncotarget, vol. 7, no. 45, pp. 73016-73031, 2016.
[22] K.-Y. Chiu, C.-C. Wu, C.-H. Chia, S.-L. Hsu, and Y.-M. Tzeng, "Inhibition of growth, migration and invasion of human bladder cancer cells by antrocin, a sesquiterpene lactone isolated from Antrodia cinnamomea, and its molecular mechanisms," Cancer Letters, vol. 373, no. 2, pp. 174-184, 2016.

[23] C.-C. Lin, C.-C. Chen, Y.-H. Kuo, J.-T. Kuo, K. J. Senthil Kumar, and S.-Y. Wang, "2,3,5-Trimethoxy-4-cresol, an anti-metastatic constituent from the solid-state cultured mycelium of Antrodia cinnamomea and its mechanism," Journal of Natural Medicines, vol. 69, no. 4, pp. 513-521, 2015.

[24] M.-K. Lu, T.-Y. Lin, C.-H. Chao, C.-H. Hu, and H.-Y. Hsu, "Molecular mechanism of Antrodia cinnamomea sulfated polysaccharide on the suppression of lung cancer cell growth and migration via induction of transforming growth factor $\beta$ receptor degradation," International Journal of Biological Macromolecules, vol. 95, pp. 1144-1152, 2017.

[25] T.-Y. Chung, F.-Y. Li, C.-I. Chang, T.-R. Jinn, and J. T. C. Tzen, "Inhibition of $\mathrm{Na}+/ \mathrm{K}+-\mathrm{ATPase}$ by antcins, unique steroid-like compounds in Antrodia camphorate," American Journal of Chinese Medicine, vol. 40, no. 5, pp. 953-965, 2012.

[26] C.-I. Lai, Y.-L. Chu, C.-T. Ho, Y.-C. Su, Y.-H. Kuo, and L.-Y. Sheen, "Antcin K, an active triterpenoid from the fruiting bodies of basswood cultivated Antrodia cinnamomea, induces mitochondria and endoplasmic reticulum stress-mediated apoptosis in human hepatoma cells," Journal of Traditional and Complementary Medicine, vol. 6, no. 1, pp. 48-56, 2016.

[27] K. B. Male, Y. K. Rao, Y.-M. Tzeng, J. Montes, A. Kamen, and J. H. T. Luong, "Probing inhibitory effects of Antrodia camphorata isolates using insect cell-based impedance spectroscopy: inhibition vs chemical structure," Chemical Research in Toxicology, vol. 21, no. 11, pp. 2127-2133, 2008.

[28] Y. K. Rao, M. Geethangili, and Y.-M. Tzeng, "Development of a high performance liquid chromatography method for the quantitative determination of bioactive triterpenoids in the extracts of Antrodia camphorata," Analytical Methods, vol. 5, no. 20, pp. 5724-5730, 2013.

[29] R. Kajanne, P. Miettinen, A. Mehlem et al., "EGF-R regulates MMP function in fibroblasts through MAPK and AP-1 pathways," Journal of Cellular Physiology, vol. 212, no. 2, pp. 489-497, 2007.

[30] H. T. Cohen and F. J. McGovern, "Renal-cell carcinoma," The New England Journal of Medicine, vol. 353, no. 23, pp. 24772490, 2005.

[31] J. Zhao and J. Guan, "Signal transduction by focal adhesion kinase in cancer," Cancer and Metastasis Reviews, vol. 28, no. 1-2, pp. 35-49, 2009.

[32] L. Li and W. Li, "Epithelial-mesenchymal transition in human cancer: comprehensive reprogramming of metabolism, epigenetics, and differentiation," Pharmacology \& Therapeutics, vol. 150, pp. 33-46, 2015.

[33] P. S. Leventhal, E. A. Shelden, B. Kim, and E. L. Feldman, “Tyrosine phosphorylation of paxillin and focal adhesion kinase during insulin-like growth factor-I-stimulated lamellipodial advance," The Journal of Biological Chemistry, vol. 272, no. 8, pp. 5214-5218, 1997.

[34] R. J. Motzer, T. E. Hutson, D. Cella et al., "Pazopanib versus sunitinib in metastatic renal-cell carcinoma," The New England Journal of Medicine, vol. 369, no. 8, pp. 722-731, 2013.

[35] A. AlQathama and J. M. Prieto, "Natural products with therapeutic potential in melanoma metastasis," Natural Product Reports, vol. 32, no. 8, pp. 1170-1182, 2015. 
[36] T. N. Chinembiri, L. H. Du Plessis, M. Gerber, J. H. Hamman, and J. Du Plessis, "Review of natural compounds for potential skin cancer treatment," Molecules, vol. 19, no. 8, pp. 11679-11721, 2014.

[37] P. Kratimenos, I. Koutroulis, D. Marconi et al., "Multi-targeted molecular therapeutic approach in aggressive neuroblastoma: The effect of Focal Adhesion Kinase-Src-Paxillin system," Expert Opinion on Therapeutic Targets, vol. 18, no. 12, pp. 13951406, 2014.

[38] C. E. Turner, "Paxillin interactions," Journal of Cell Science, vol. 113, part 23, pp. 4139-4140, 2000.

[39] T. S. Panetti, "Tyrosine phosphorylation of paxillin, FAK, and p130CAS: effects on cell spreading and migration," Frontiers in Bioscience, vol. 7, pp. d143-d150, 2002.

[40] N. A. Chatzizacharias, G. P. Kouraklis, and S. E. Theocharis, "Clinical significance of FAK expression in human neoplasia," Histology and Histopathology, vol. 23, no. 5, pp. 629-650, 2008.

[41] M. Luo, H. Fan, T. Nagy et al., "Mammary epithelial-specific ablation of the focal adhesion kinase suppresses mammary tumorigenesis by affecting mammary cancer stem/progenitor cells," Cancer Research, vol. 69, no. 2, pp. 466-474, 2009.

[42] M. J. van Nimwegen and B. van de Water, "Focal adhesion kinase: a potential target in cancer therapy," Biochemical Pharmacology, vol. 73, no. 5, pp. 597-609, 2007.

[43] F. Dai, Y. Chen, L. Huang et al., "A novel synthetic small molecule YH-306 suppresses colorectal tumour growth and metastasis via FAK pathway," Journal of Cellular and Molecular Medicine, vol. 19, no. 2, pp. 383-395, 2015.

[44] L. Bai, J. C. Yang, J.-H. Ok, P. C. MacK, H.-J. Kung, and C. P. Evans, "Simultaneous targeting of Src kinase and receptor tyrosine kinase results in synergistic inhibition of renal cell carcinoma proliferation and migration," International Journal of Cancer, vol. 130, no. 11, pp. 2693-2702, 2012.

[45] L. Rice, S. Lepler, C. Pampo, and D. W. Siemann, "Impact of the SRC inhibitor dasatinib on the metastatic phenotype of human prostate cancer cells," Clinical \& Experimental Metastasis, vol. 29, no. 2, pp. 133-142, 2012.

[46] J.-Y. Chen, Y.-A. Tang, S.-M. Huang et al., "A novel sialyltransferase inhibitor suppresses FAK/paxillin signaling and cancer angiogenesis and metastasis pathways," Cancer Research, vol. 71, no. 2, pp. 473-483, 2011.

[47] C. Ballestrem, B. Wehrle-Haller, B. Hinz, and B. A. Imhof, "Actin-dependent lamellipodia formation and microtubuledependent tail retraction control-directed cell migration," Molecular Biology of the Cell (MBoC), vol. 11, no. 9, pp. 29993012, 2000.

[48] D. C. Radisky and M. A. LaBarge, "Epithelial-mesenchymal transition and the stem cell phenotype," Cell Stem Cell, vol. 2, no. 6, pp. 511-512, 2008.

[49] D. Nojima, K. Nakajima, L. Li et al., "CpG methylation of promoter region inactivates E-cadherin gene in renal cell carcinoma," Molecular Carcinogenesis, vol. 32, no. 1, pp. 19-27, 2001.

[50] A. Katagiri, R. Watanabe, and Y. Tomita, "E-cadherin expression in renal cell cancer and its significance in metastasis and survival," British Journal of Cancer, vol. 71, no. 2, pp. 376-379, 1995.

[51] M. Schoumacher, R. D. Goldman, D. Louvard, and D. M. Vignjevic, "Actin, microtubules, and vimentin intermediate filaments cooperate for elongation of invadopodia," The Journal of Cell Biology, vol. 189, no. 3, pp. 541-556, 2010.
[52] M. Wu, X. Bai, G. Xu et al., "Proteome analysis of human androgen-independent prostate cancer cell lines: variable metastatic potentials correlated with vimentin expression," Proteomics, vol. 7, no. 12, pp. 1973-1983, 2007.

[53] M. Ii, H. Yamamoto, Y. Adachi, Y. Maruyama, and Y. Shinomura, "Role of matrix metalloproteinase-7 (matrilysin) in human cancer invasion, apoptosis, growth, and angiogenesis," Experimental Biology and Medicine, vol. 231, no. 1, pp. 20-27, 2006.

[54] M. Lein, K. Jung, C. Laube et al., "Matrix-metalloproteinases and their inhibitors in plasma and tumor tissue of patients with renal cell carcinoma," International Journal of Cancer, vol. 85, no. 6, pp. 801-804, 2000.

[55] Y. Gong, U. D. Chippada-Venkata, and W. K. Oh, "Roles of matrix metalloproteinases and their natural inhibitors in prostate cancer progression," Cancers, vol. 6, no. 3, pp. 12981327, 2014.

[56] K. Brew and H. Nagase, "The tissue inhibitors of metalloproteinases (TIMPs): an ancient family with structural and functional diversity," Biochimica et Biophysica Acta (BBA)Molecular Cell Research, vol. 1803, no. 1, pp. 55-71, 2010.

[57] A. H. Baker, S. J. George, A. B. Zaltsman, G. Murphy, and A. C. Newby, "Inhibition of invasion and induction of apoptotic cell death of cancer cell lines by overexpression of TIMP-3," British Journal of Cancer, vol. 79, no. 9-10, pp. 1347-1355, 1999.

[58] S. S. Apte, B. R. Olsen, and G. Murphy, "The gene structure of tissue inhibitor of metalloproteinases (TIMP)-3 and its inhibitory activities define the distinct TIMP gene family," The Journal of Biological Chemistry, vol. 270, no. 24, pp. 14313-14318, 1995.

[59] M. Bodnar, L. Szylberg, W. Kazmierczak, and A. Marszalek, "Tumor progression driven by pathways activating matrix metalloproteinases and their inhibitors," Journal of Oral Pathology \& Medicine, vol. 44, no. 6, pp. 437-443, 2015.

[60] H. Yoshiji, S. R. Harris, E. Raso et al., "Mammary carcinoma cells over-expressing tissue inhibitor of metalloproteinases-1 show enhanced vascular endothelial growth factor expression," International Journal of Cancer, vol. 75, no. 1, pp. 81-87, 1998.

[61] H.-J. Kim, Y.-R. Cho, S. H. Kim, and D.-W. Seo, “TIMP-2derived 18-mer peptide inhibits endothelial cell proliferation and migration through cAMP/PKA-dependent mechanism," Cancer Letters, vol. 343, no. 2, pp. 210-216, 2014.

[62] D.-W. Seo, H. Li, L. Guedez et al., "TIMP-2 mediated inhibition of angiogenesis: an MMP-independent mechanism," Cell, vol. 114, no. 2, pp. 171-180, 2003.

[63] C. A. Fernandez, R. Roy, S. Lee et al., "The anti-angiogenic peptide, Loop 6, binds insulin-like growth factor-1 receptor," The Journal of Biological Chemistry, vol. 285, no. 53, pp. 4188641895, 2010.

[64] M. Boufraqech, L. Zhang, N. Nilubol et al., "Lysyl Oxidase (LOX) transcriptionally regulates SNAI2 expression and TIMP4 secretion in human cancers," Clinical Cancer Research, vol. 22, no. 17, pp. 4491-4504, 2016.

[65] W. G. Stetler-Stevenson, "Tissue inhibitors of metalloproteinases in cell signaling: metalloproteinase-independent biological activities," Science Signaling, vol. 1, re6, no. 27, 2008.

[66] J. H. Qi, Q. Ebrahem, N. Moore et al., "A novel function for tissue inhibitor of metalloproteinases-3 (TIMP3): inhibition of angiogenesis by blockage of VEGF binding to VEGF receptor2," Nature Medicine, vol. 9, no. 4, pp. 407-415, 2003.

[67] K.-H. Kang, S.-Y. Park, S. B. Rho, and J.-H. Lee, "Tissue inhibitor of metalloproteinases-3 interacts with angiotensin II 
type 2 receptor and additively inhibits angiogenesis," Cardiovascular Research, vol. 79, no. 1, pp. 150-160, 2008.

[68] Y.-J. Chen, Y.-Y. Wei, H.-T. Chen et al., "Osteopontin increases migration and MMP-9 up-regulation via $\alpha \mathrm{v} \beta 3$ integrin, FAK, ERK, and NF- $\kappa$ B-dependent pathway in human chondrosarcoma cells," Journal of Cellular Physiology, vol. 221, no. 1, pp. 98$108,2009$.

[69] H.-Y. Xu, A.-R. Qian, P. Shang et al., "siRNA targeted against HAb18G/CD147 inhibits MMP-2 secretion, actin and FAK expression in hepatocellular carcinoma cell line via ERK1/2 pathway," Cancer Letters, vol. 247, no. 2, pp. 336-344, 2007.

[70] H.-J. Cho, J. H. Kang, J.-Y. Kwak et al., "Ascofuranone suppresses PMA-mediated matrix metalloproteinase-9 gene activation through the Ras/Raf/MEK/ERK- and Apl-dependent mechanisms," Carcinogenesis, vol. 28, no. 5, pp. 1104-1110, 2007.

[71] H. Han, B. Du, X. Pan et al., "CADPE inhibits PMA-stimulated gastric carcinoma cell invasion and matrix metalloproteinase9 expression by FAK/MEK/ERK-mediated AP-1 activation," Molecular Cancer Research, vol. 8, no. 11, pp. 1477-1488, 2010.

[72] P. Verde, L. Casalino, F. Talotta, M. Yaniv, and J. B. Weitzman, "Deciphering AP-1 function in tumorigenesis: Fra-ternizing on target promoters," Cell Cycle, vol. 6, no. 21, pp. 2633-2639, 2007.

[73] Y.-C. Tu, D.-Y. Huang, S.-G. Shiah, J.-S. Wang, and W.-W. Lin, "Regulation of c-Fos gene expression by NF- $\kappa$ B: A p65 homodimer binding site in mouse embryonic fibroblasts but not human HEK293 cells," PLoS ONE, vol. 8, no. 12, Article ID e84062, 2013.

[74] M. C. Chang, C. A. Chen, P. J. Chen et al., "Mesothelin enhances invasion of ovarian cancer by inducing MMP-7 through MAPK/ERK and JNK pathways," Biochemical Journal, vol. 442, no. 2, pp. 293-302, 2012.

[75] B.-Y. Ho, Y.-M. Wu, K.-J. Chang, and T.-M. Pan, "Dimerumic acid inhibits SW620 cell invasion by attenuating $\mathrm{H}_{2} \mathrm{O}_{2}$ mediated MMP-7 expression via JNK/C-Jun and ERK/C-Fos activation in an AP-1-Dependent manner," International Journal of Biological Sciences, vol. 7, no. 6, pp. 869-880, 2011.

[76] Z.-C. Jia, Y.-L. Wan, J.-Q. Tang et al., “Tissue factor/activated factor VIIa induces matrix metalloproteinase-7 expression through activation of c-Fos via ERK1/2 and p38 MAPK signaling pathways in human colon cancer cell," International Journal of Colorectal Disease, vol. 27, no. 4, pp. 437-445, 2012.

[77] D. M. Cortez, M. D. Feldman, S. Mummidi et al., "IL-17 stimulates MMP-1 expression in primary human cardiac fibroblasts via 338 MAPK- and ERK1/2-dependent C/EBP- $\beta$, NF- $\kappa$ B, and AP-1 activation," American Journal of Physiology-Heart and Circulatory Physiology, vol. 293, no. 6, pp. H3356-H3365, 2007.

[78] D. A. Armstrong, L. N. Phelps, and M. P. Vincenti, "CCAAT enhancer binding protein $\beta$ regulates matrix metalloproteinase-1 expression in interleukin-1 $\beta$-stimulated A549 lung carcinoma cells," Molecular Cancer Research, vol. 7, no. 9, pp. 1517-1524, 2009.

[79] G. Buttice, M. Duterque-Coquillaud, J. P. Basuyaux, S. Carrere, M. Kurkinen, and D. Stehelin, "Erg, an Ets-family member, differentially regulates human collagenasel (MMP1) and stromelysin1 (MMP3) gene expression by physically interacting with the Fos/Jun complex," Oncogene, vol. 13, pp. 2297-2306, 1996.

[80] Y. Fei, E. Shimizu, M. W. McBurney, and N. C. Partridge, "Sirtuin 1 is a negative regulator of parathyroid hormone stimulation of matrix metalloproteinase 13 expression in osteoblastic cells: role of sirtuin 1 in the action of PTH on osteoblasts," The
Journal of Biological Chemistry, vol. 290, no. 13, pp. 8373-8382, 2015.

[81] Y. Luo, F. Liang, and Z.-Y. Zhang, "PRL1 promotes cell migration and invasion by increasing MMP2 and MMP9 expression through Src and ERK1/2 pathways," Biochemistry, vol. 48, no. 8, pp. 1838-1846, 2009.

[82] T. Okuma, M. Hirata, F. Yano et al., "Regulation of mouse chondrocyte differentiation by CCAAT/enhancer-binding proteins," Biomedical Research (Japan), vol. 36, no. 1, pp. 21-29, 2015. 


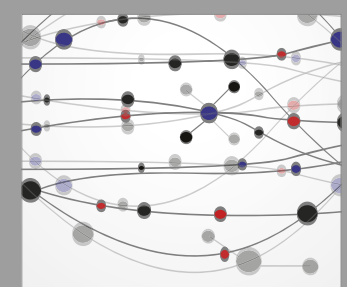

The Scientific World Journal
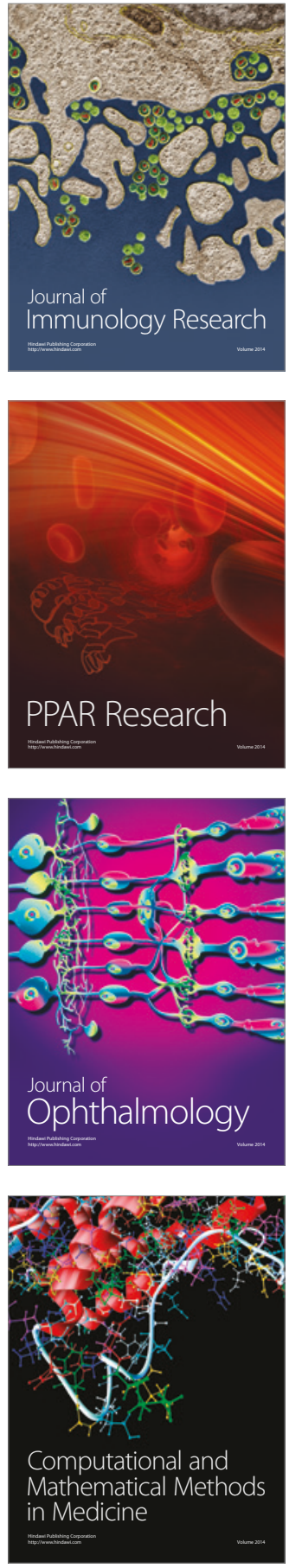

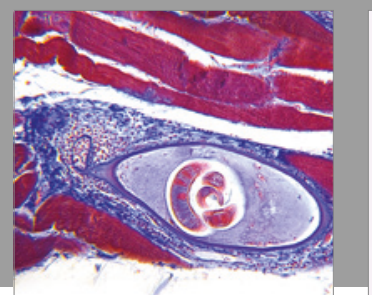

Gastroenterology Research and Practice
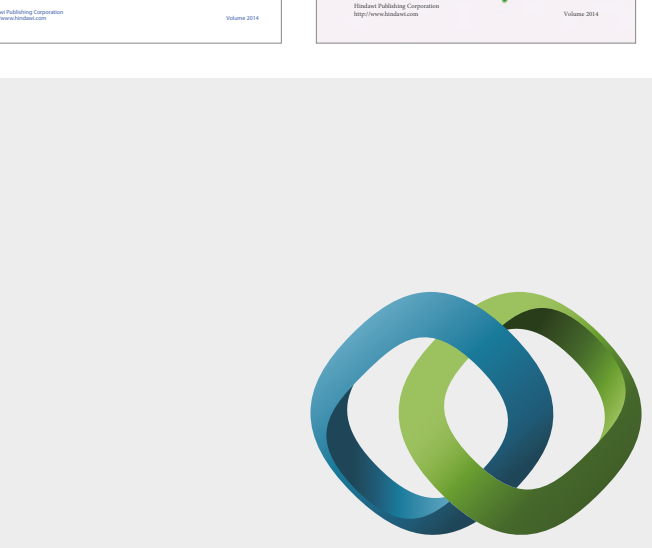

\section{Hindawi}

Submit your manuscripts at

https://www.hindawi.com
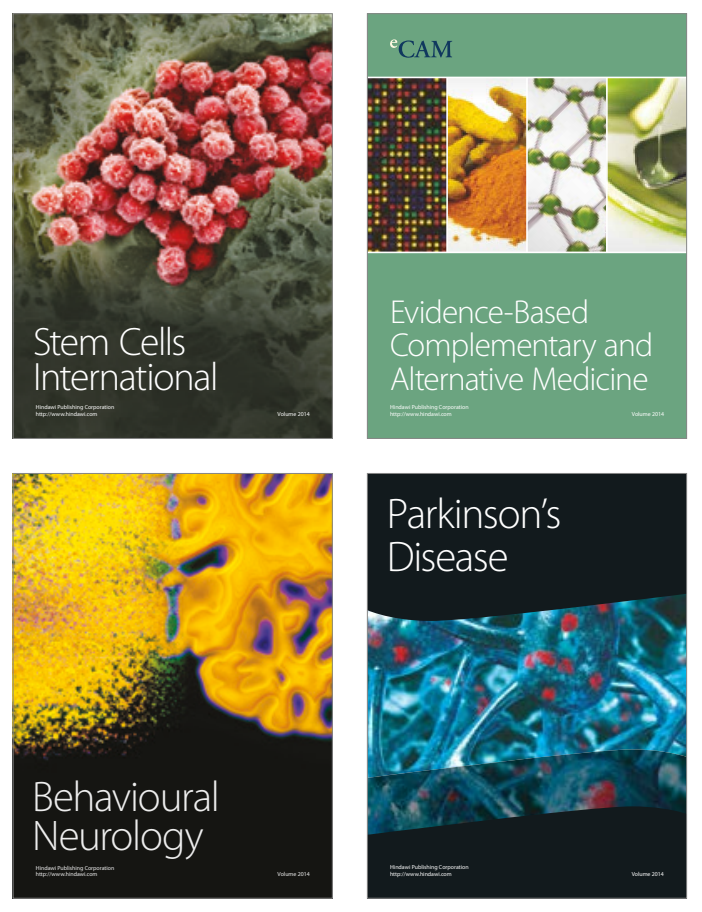
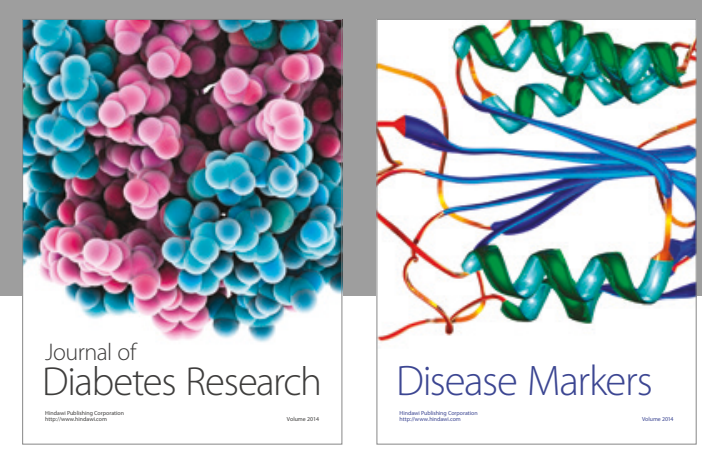

Disease Markers
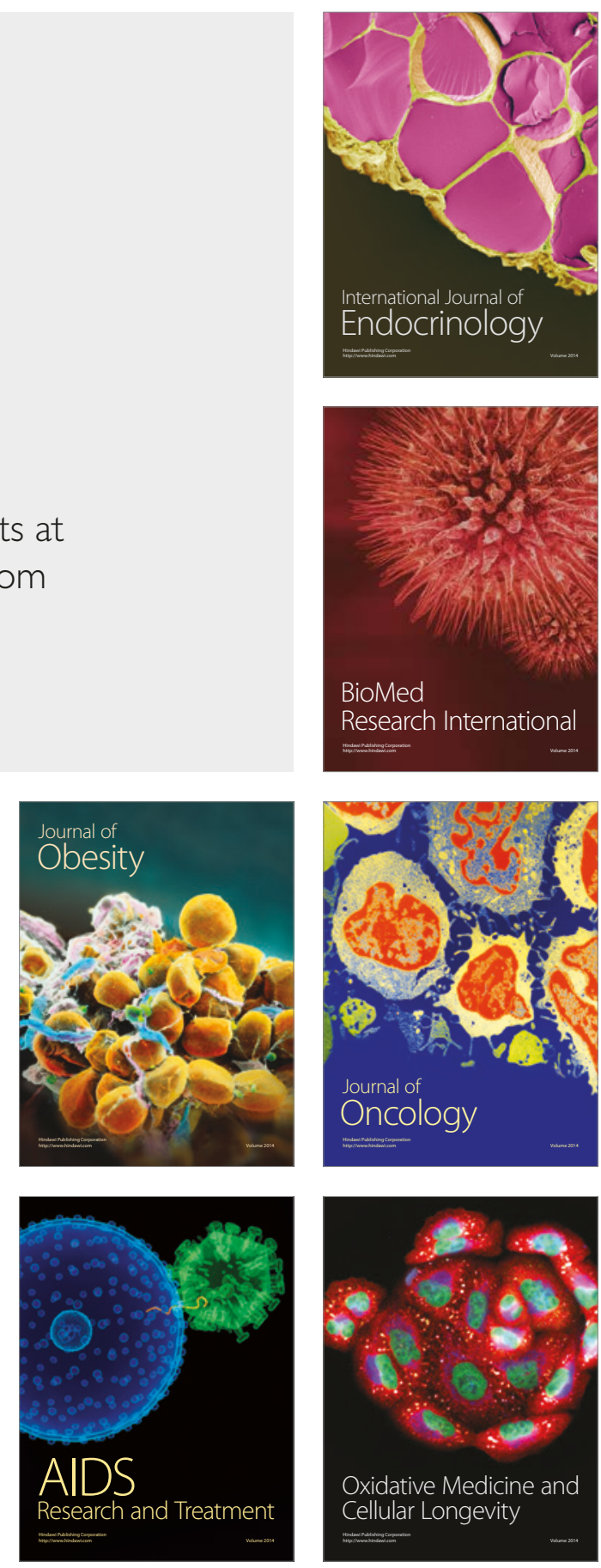